\title{
On KP-integrable Hurwitz functions
}

\author{
A. Alexandrov,,$^{a, b, c}$ A. Mironov, ${ }^{d, c, e}$ A. Morozov ${ }^{c, e}$ and S. Natanzon ${ }^{f, g}$ \\ ${ }^{a}$ Freiburg Institute for Advanced Studies (FRIAS), University of Freiburg, \\ Albertstraße 19, D-79104 Freiburg, Germany \\ ${ }^{b}$ Mathematics Institute, University of Freiburg, \\ Eckerstraße 1, D-79104 Freiburg, Germany \\ ${ }^{c}$ ITEP, \\ Bol. Cheremushkinskaya 25, Moscow 117218, Russia \\ ${ }^{d}$ Theory Department, Lebedev Physics Institute, \\ Leninsky 53, Moscow 119991, Russia \\ ${ }^{e}$ National Research Nuclear University MEPhI, \\ Kashirskoe sh. 31, Moscow 115409, Russia \\ ${ }^{f}$ National Research University Higher School of Economics, \\ Vavilova st. 7a, Moscow, Russia \\ ${ }^{g}$ Laboratory of Quantum Topology, Chelyabinsk State University, \\ Brat'ev Kashirinykh street 129, Chelyabinsk 454001, Russia \\ E-mail: alexandrovsash@gmail.com, mironov@lpi.ru, morozov@itep.ru, \\ natanzons@mail.ru
}

ABSTRACT: There is now a renewed interest [1]-[4] to a Hurwitz $\tau$-function, counting the isomorphism classes of Belyi pairs, arising in the study of equilateral triangulations and Grothiendicks's dessins d'enfant. It is distinguished by belonging to a particular family of Hurwitz $\tau$-functions, possessing conventional Toda/KP integrability properties. We explain how the variety of recent observations about this function fits into the general theory of matrix model $\tau$-functions. All such quantities possess a number of different descriptions, related in a standard way: these include Toda/KP integrability, several kinds of $W$-representations (we describe four), two kinds of integral (multi-matrix model) descriptions (of Hermitian and Kontsevich types), Virasoro constraints, character expansion, embedding into generic set of Hurwitz $\tau$-functions and relation to knot theory. When approached in this way, the family of models in the literature has a natural extension, and additional integrability with respect to associated new time-variables. Another member of this extended family is the Itsykson-Zuber integral.

Keywords: Matrix Models, Integrable Hierarchies, Topological Field Theories

ARXIV EPRINT: 1405.1395 


\section{Contents}

1 Introduction 1

2 Representation via cut-and-join operators 5

$\begin{array}{llr}3 & \text { Representation via Casimir operators } & 6\end{array}$

$4 Z_{(2, n)}$ as a $\tau$-function of Toda lattice $\quad 8$

5 Matrix model representations $\quad 10$

6 Miwa transformation to Kontsevich matrix models 12

7 Virasoro/ $\widetilde{W}$ constraints $\quad 14$

8 Naive $W$-representations $\quad 16$

8.1 The case of $Z_{(1,1)} 16$

$\begin{array}{ll}8.2 \text { Direct check of }(8.12) & 17\end{array}$

$\begin{array}{ll}8.3 \text { The case of } Z_{(1,2)} & 18\end{array}$

$\begin{array}{lll}8.4 \text { Operators } \hat{\mathcal{O}}\left(u_{1}, \ldots, u_{n}\right) & 19\end{array}$

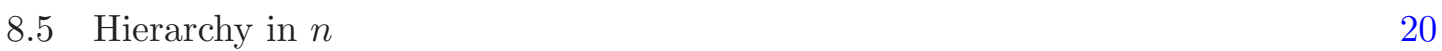

9 Description in terms of the $w_{\infty}$-algebra $\quad 20$

9.1 Combined Casimir operators $\hat{\widetilde{C}}$ as distinguished $\hat{W}_{0}^{(m)} \quad 20$

$\begin{array}{lll}9.2 & \text { Relation between the two } W \text {-representations } & 22\end{array}$

9.3 More details from the $w_{\infty}$ dictionary 23

10 Conclusion $\quad 24$

\section{Introduction}

Hurwitz $\tau$-function [5-8] is a new important subject of theoretical physics, which seems relevant to description of non-perturbative phenomena beyond $2 d$ conformal field theory, actually beginning from the $3 d$ Chern-Simons and knot theory, see [9-11]. In general, Hurwitz $\tau$-functions do not belong $[7,8]$ to a narrower well-studied class of $\mathrm{KP} /$ Toda $\tau$-functions, i.e. are not straightforwardly reducible to free fermions $(\widehat{\mathrm{U}(1)}$ Kac-Moody algebras) and Plucker relations (the Universal Grassmannian). However, the special cases, when they $d o$, help to establish links between the known and unknown, and are very instructive for development of terminology and research directions. A particular case of the previously known example of this type $[12,13]$ was recently considered again in [1]-[4] 
and finally seems to attract reasonable attention. In the present paper we further extend it and consider from the perspective of the modern $\tau$-function theory, thus slightly broadening the consideration in those papers.

In systematic presentation, the story begins from the celebrated formula [14] for the Hurwitz numbers,

$$
\mathcal{N}_{\Delta_{1}, \ldots, \Delta_{k}}=\sum_{R} d_{R}^{2} \varphi_{R}\left(\Delta_{1}\right) \ldots \varphi_{R}\left(\Delta_{k}\right)
$$

which expresses them through the properly normalized symmetric-group characters $\varphi_{R}(\Delta)$. Here $\Delta_{1}, \ldots, \Delta_{k}$ and $R$ are Young diagrams and $d_{R}$ is the dimension of representation $R$ of the symmetric group $S_{|R|}$ divided by $|R|$ !, [15-18]. The ordinary Hurwitz numbers (counting ramified coverings of the Riemann sphere with ramifications of a given type) arise when all $\Delta_{1}, \ldots, \Delta_{k}$ have the same size (the same number of boxes), then the sum in (1.1) goes over $R$ of the same size. If the size $|\Delta|>|R|$, then $\varphi_{R}(\Delta)=0$, if $|\Delta|<|R|$, then

$$
\varphi_{R}(\Delta)=\frac{(|R|-|\Delta|+k) !}{k !(|R|-|\Delta|) !} \varphi_{R}\left(\Delta, 1^{|R|-|\Delta|}\right)
$$

where at the r.h.s. $|R|-|\Delta|$ lines of unit length is added to the Young diagram $\Delta$, and $k$ is the number of lines of unit length in the diagram $\Delta$. See $[5,6,19]$ and especially $[7,8]$ for more details about all this.

The symmetric group characters $\varphi_{R}(\Delta)$ are related to the linear group ones (the Schur functions)

as follows $[15-18]$

$$
\chi_{R}[X]=\left.\chi_{R}\{p\}\right|_{p_{n}=\operatorname{Tr} X^{n}}
$$

$$
\chi_{R}\{p\}=\sum_{\Delta} d_{R} \varphi_{R}(\Delta) p_{\Delta} \cdot \delta_{|R|,|\Delta|}
$$

or $[19]$

$$
\chi_{R}\left\{p_{m}+\delta_{m, 1}\right\}=\sum_{\Delta} d_{R} \varphi_{R}(\Delta) p_{\Delta}
$$

The difference between the two is that in (1.4) the sum goes only over $|\Delta|$ of size $|R|$, while in (1.5) there is no restriction. For a Young diagram $\Delta:=\delta_{1} \geq \delta_{2} \geq \ldots \geq \delta_{l(\Delta)}$, which is an ordered partition of $|\Delta|$ into a sum of $l(\Delta)$ integers $\delta_{i}$, associated is the multi-time variable

$$
p_{\Delta}=p_{\delta_{1}} p_{\delta_{2}} \ldots p_{\delta_{l(\Delta)}}
$$

In the particular case when all $p_{n}$ are the same, $p_{n}=N$, i.e. when $X$ is an $N \times N$ unit matrix, $X=I_{N}$, eq. (1.4) provides $\varphi$-decomposition of the dimensions $D_{R}(N)$ of the irreducible representation $R$ of the Lie algebra $g l(N)$

$$
D_{R}(N)=\chi_{R}\left[I_{N}\right]=\sum_{\Delta} d_{R} \varphi_{R}(\Delta) N^{l(\Delta)} \delta_{|R|,|\Delta|}
$$

The standard definition of these dimensions is the celebrated hook formula $[15-18]$

$$
\frac{D_{R}(N)}{d_{R}}=\prod_{i, j \in R}(N+i-j)=\prod_{i} \frac{\left(\lambda_{i}+N-i\right) !}{(N-i) !}
$$


In fact, for study of integrability important is just the fact that all $p_{n}$ are the same, and in what follows we mostly use the letter $u$ instead of $N$, to downplay association with the representation dimensions and emphasize that $u$ does not need to be a positive integer.

Combining (1.1) and (1.4), it is natural to consider the generating function ${ }^{1}$

$$
h_{k}\left\{p^{(1)}, \ldots, p^{(k)}\right\}=\sum_{\Delta_{1}, \ldots, \Delta_{k}} \mathcal{N}_{\Delta_{1}, \ldots, \Delta_{k}} p_{\Delta_{1}} \ldots p_{\Delta_{k}}=\sum_{R} d_{R}^{2} \prod_{i=1}^{k} \frac{\chi_{R}\left\{p^{(i)}\right\}}{d_{R}}
$$

It is well known that for $k=1$ and $k=2$ these $h$-functions are KP and Toda lattice $\tau$-functions respectively; moreover, they are trivial $\tau$-functions:

$$
\begin{aligned}
h_{1}\{p\} & =\sum_{R} d_{R} \chi_{R}\{p\}=e^{p_{1}}, \\
h_{2}\{\bar{p}, p\} & =\sum_{R} \chi_{R}\{\bar{p}\} \chi_{R}\{p\}=\exp \left(\sum_{m} \frac{1}{m} \bar{p}_{m} p_{m}\right)
\end{aligned}
$$

It is also known $[7,8]$ that for $k \geq 3$ with generic $p^{(i \geq 3)}$ these $h$-functions do not belong to the $\mathrm{KP} /$ Toda family as functions of $\left\{p^{(1)}\right\}$ or $\left\{p^{(1)}, p^{(2)}\right\}$. However, of course, this can happen for particular choices of $\left\{p^{(i \geq 3)}\right\}$, and they $d o$, provided all $p_{m}^{(i)}=u^{(i)}$ for all $m$.

In other words, making use of (1.7) we restrict $h$-functions to more specific generating functions:

$$
Z_{(k, n)}\left(s, u_{1}, \ldots, u_{n} \mid p^{(i)}\right)=\sum_{R} s^{|R|} d_{R}^{2-k-n}\left(\prod_{i=1}^{k} \chi_{R}\left\{p^{(i)}\right\}\right)\left(\prod_{i=1}^{n} D_{R}\left(u_{i}\right)\right)
$$

at $k=1,2$, which, given their origin and properties, we call hypergeometric (following [12, 13]) Hurwitz $\tau$-functions. The formally continued to negative values $(2,-1)$ member of this family $Z_{(2,-1)}$ is the celebrated Itsykson-Zuber integral:

$$
Z_{(2,-1)}\{\bar{p}, p\}=\sum_{R} \frac{d_{R} \chi_{R}[X] \chi_{R}[Y]}{D_{R}(N)}=J_{I Z}(N)
$$

with $p_{n}=\operatorname{tr} X^{n}$ and $\bar{p}_{n}=\operatorname{tr} Y^{n}$ (see eq. (77) in [20]), note that for representations $R$ with $D_{R}(N)=0$ the characters in the numerator are also vanishing, and these $R$ do not contribute to the sum. For $(1,0)$ and $(1,1)$ we get just the trivial exponentials

$$
Z_{(1,0)}=\sum_{R} s^{|R|} d_{R} \chi_{R}\{p\}=e^{s p_{1}}
$$

and

$$
Z_{(1,1)}=\sum_{R} s^{|R|} D_{R}(N) \chi_{R}\{p\}=\exp \left(N \sum_{m=1}^{N} \frac{s^{m} p_{m}}{m}\right)
$$

\footnotetext{
${ }^{1}$ This definition could depend slightly on whether one imposes restrictions like $\left|\Delta_{i}\right|=\left|\Delta_{j}\right|$ and $|R|=\left|\Delta_{i}\right|$ in the sums.
} 
The particular case $Z_{(1,2)}$ of generating numbers of isomorphism classes of the Belyi pairs was studied in $[2-4] .^{2}$

In fact, models $Z_{(1, n)}$ with $n>2$ are far more interesting. This becomes obvious already for $N=1$, when only symmetric diagrams $R=[m]$ contribute, with $D_{[m]}(N=1)=1$ and $d_{[m]}=1 / m$ !, so that (1.11) turns into a simple series

$$
Z_{(1, n)}\left(\text { all } u_{i}=1\right)=\sum_{m=0}^{\infty} s^{m} \chi_{[m]}\{p\} d_{[m]}^{1-n} D_{[k]}^{n}=\sum_{m=0}^{\infty}(m !)^{n-1} s^{m} \chi_{[m]}\{p\}=\sum_{m=0}^{\infty} \frac{(m !)^{n-2}\left(s p_{1}\right)^{m}}{}+O\left(p_{2}, \ldots\right)
$$

The underlined series is nicely convergent for $n=1$ and $n=2$, while for $n>2$ it is asymptotic series, defined up to non-perturbative corrections. For $n=3$ we get the archetypical example:

$$
\sum_{m} m ! \cdot s^{m}
$$

where non-perturbative ambiguity is proportional to

$$
\oint \frac{e^{-x} d x}{1-x s}=\frac{e^{-1 / s}}{s}
$$

This example appears in the study of $Z_{(1,3)}$. The usual way to handle the series like (1.15) is the integral transformation:

$$
f(s)=\sum_{m} a_{m} s^{m} \longrightarrow F(s)=\sum_{m} a_{m} m ! \cdot s^{m}=\frac{1}{s} \int_{x_{+}} e^{-x / s} f(x) d x
$$

For generic $N$ this formalism turns into the theory of Kontsevich-like models.

Of course, (1.11) are very special, besides they are $\tau$-functions $[1,12,13]$, they actually belong to the class of matrix model $\tau$-functions [45-51]. This not-yet-rigourously-defined class is characterized by coexistence of a wide variety of very different representations and properties [52]:

\footnotetext{
${ }^{2}$ Belyi pair describes a complex curve as a covering of $C P^{1}$, ramified at just three points $0,1, \infty$ (the pair is the curve $C$ and the mapping $C \longrightarrow C P^{1}$ ). According to G.Belyi and A.Grothendieck [21-25], existence of such representation is necessary and sufficient for arithmeticity of the curve and arithmetic curves are in one-to-one correspondence with the equilateral triangulations (dessins d'enfant). Thus, enumeration of Belyi pairs is a typical matrix model problem (see more on relations between counting the Belyi maps, Hurwitz numbers and matrix models in [26-28]), though equivalence of matrix model [29, 30] and sumover-metrics descriptions [31, 32], proved in [33, 34] on the lines of [35-41] remains a big mystery from the point of view of the complicated embedding of moduli space of arithmetic curves into the entire moduli space, see [42] and, for a related consideration, [43]. The Belyi pairs are enumerated by the triple Hurwitz numbers $\mathcal{N}_{\Delta_{0}, \Delta_{1}, \Delta_{\infty}}$, but no adequate language is still found to describe the full generating function $h_{3}\left\{p^{(1)}, p^{(2)}, p^{(3)}\right)=\sum_{R} d_{R}^{-1} \chi_{R}\left(p^{(1)}\right) \chi_{R}\left(p^{(2)}\right) \chi_{R}\left(p^{(3)}\right)$, see [7, 8]. The suggestion of [2] was to sacrifice any details about $\Delta_{0}$ and $\Delta_{1}$ and keep only information about the numbers $l\left(\Delta_{0}\right)$ and $l\left(\Delta_{1}\right)$ of unglued sheets of the covering over 0 and 1: then such special generating function $Z_{(1,2)}$ is obviously a $\mathrm{KP} \tau$-function. In fact, it is enough to do so just at one (not obligatory two) of the three points: $Z_{(2,1)}$ is also a conventional Toda lattice $\tau$-function. Presentation of standard results about these quantities and their multi-point counterparts is the purpose of the present paper. As to triple coverings, enumeration is the simplest, but not the most interesting part of the story. An explicit construction of the Belyi functions is extremely hard: for relatively vast set of examples see [44]. A crucial problem of string theory remains expressing the Mumford measure and its constituents (determinants of $\bar{\partial}$ operators) for arithmetic curves through combinatorial triple $\Delta_{1}, \Delta_{2}, \Delta_{3}$.
} 
- they are KP/Toda $\tau$-functions,

- they possess integral ("matrix-model") representations of "ordinary" and Kontsevich types,

- they satisfy Virasoro- or W-like constraints (possess a $D$-module representation and obey the AMM/EO topological recursion [53-61]),

- they possess various $W$-representations [62-64], including ones via Casimir operators and via cut-and-join operators,

- they possess special linear decompositions into linear- and symmetric-group characters,

- they are Hurwitz $\tau$-functions.

The purpose of this paper is to describe all these properties within the context of the hypergeometric Hurwitz $\tau$-functions (1.11).

For illustrative purposes and to avoid notational confusions we list the simplest examples of dimensions (1.8), linear group characters $\chi_{R}\{p\}$, and appropriately normalized symmetric group characters $\varphi_{R}(\Delta)$ from $[5,6]$ :

\begin{tabular}{|c|c|c|c|c|c|c|c|c|c|c|}
\hline$R$ & $D_{R}(N) / d_{R}$ & $\chi_{R}\{p\}$ & $d_{R}$ & $\varphi_{R}(1)$ & $\varphi_{R}(2)$ & $\varphi_{R}(11)$ & $\varphi_{R}(3)$ & $\varphi_{R}(21)$ & $\varphi_{R}(111)$ & \\
\hline$[1]$ & $N$ & $p_{1}$ & 1 & 1 & & & & & & \\
\hline$[2]$ & $N(N+1)$ & $\frac{p_{2}+p_{1}^{2}}{2}$ & $\frac{1}{2}$ & 2 & 1 & 1 & & & & \\
\hline [11] & $N(N-1)$ & $\frac{-p_{2}+p_{1}^{2}}{2}$ & $\frac{1}{2}$ & 2 & -1 & 1 & & & & \\
\hline$[3]$ & $N(N+1)(N+2)$ & $\frac{2 p_{3}+3 p_{2} p_{1}+p_{1}^{3}}{6}$ & $\frac{1}{6}$ & 3 & 3 & 3 & 2 & 3 & 1 & \\
\hline$[21]$ & $(N-1) N(N+1)$ & $\frac{-p_{3}+p_{1}^{3}}{3}$ & $\frac{1}{3}$ & 3 & 0 & 3 & -1 & 0 & 1 & \\
\hline$[111]$ & $N(N-1)(N-2)$ & $\frac{2 p_{3}-3 p_{2} p_{1}+p_{1}^{3}}{6}$ & $\frac{1}{6}$ & 3 & -3 & 3 & 2 & -3 & 1 & \\
\hline
\end{tabular}

\section{Representation via cut-and-join operators}

The linear group characters (Schur functions) $\chi_{R}\{p\}$ are common eigenfunctions of the set of commuting generalized cut-and-join operators [5,6], and symmetric group characters $\varphi_{R}(\Delta)$ are their corresponding eigenvalues:

$$
\hat{W}_{\Delta \chi_{R}}=\varphi_{R}(\Delta) \chi_{R}
$$

What we need in (1.11) are rather operators with slightly different eigenvalues:

$$
\hat{O}(u) \chi_{R}=\frac{D_{R}(u)}{d_{R}} \chi_{R}
$$

However, eq. (1.7) allows one to make them easily from $\hat{W}_{\Delta}$ :

$$
\hat{O}(u)=\sum_{\Delta} u^{l(\Delta)} \hat{W}_{\Delta} \hat{P}_{|\Delta|}
$$


where $\hat{P}_{|\Delta|}$ is a projector, selecting the Young diagrams of the size $|\Delta|$,

$$
\hat{P}_{|\Delta|}=\oint \frac{d z}{z} z^{-|\Delta|+\hat{L}_{0}}
$$

with

$$
\hat{L}_{0}=\sum_{n} n p_{n} \frac{\partial}{\partial p_{n}}
$$

so that

$$
\hat{P}_{|\Delta| \chi_{R}}=\chi_{R} \delta_{|R|,|\Delta|}
$$

and $\hat{W}_{\Delta}$ are the general cut-and-join operators from $[5,6]$.

Thus

$$
\begin{aligned}
Z_{(1, n)}\left(s, u_{1}, \ldots, u_{n} \mid p\right) & =\left(\prod_{i=1}^{k} \hat{O}\left(u_{i}\right)\right) e^{s p_{1}} \\
Z_{(2, n)}\left(s, u_{1}, \ldots, u_{n} \mid \bar{p}, p\right) & =\left(\prod_{i=1}^{n} \hat{O}\left(u_{i}\right)\right) \exp \left(\sum_{m} \frac{s^{m}}{m} p_{m} \bar{p}_{m}\right)
\end{aligned}
$$

These are actually the $W$-representations [62-64] of the $\tau$-functions (1.11), because $\hat{O}(u)$ are, in fact, elements of the integrability-preserving $G L(\infty)$ group. However, this is not quite so obvious: operator (2.3) does not have a form where this property is obvious. In fact, one can make a triangular transformation in $(2.3)$ and get rid of projector operators $\hat{P}_{|\Delta|}$ :

$$
\hat{O}(u)=u^{\hat{L}_{0}}\left(1+\frac{\hat{W}_{2}}{u}+\frac{\hat{W}_{3}+\hat{W}_{22}}{u^{2}}+\frac{\hat{W}_{4}+\hat{W}_{32}+\hat{W}_{222}}{u^{3}}+\frac{\hat{W}_{5}+\hat{W}_{42}+\hat{W}_{33}+\hat{W}_{322}+\hat{W}_{2222}}{u^{4}} \ldots\right)=
$$

or

$$
\hat{O}(u)=u^{\hat{W}_{1}} \sum_{\Delta}^{\prime} u^{l(\Delta)-|\Delta|} \hat{W}_{\Delta}
$$

where sum goes over all diagrams containing no lines of unit length (we denote this restriction by prime).

Since, say $[5,6], \hat{W}_{22}=\frac{1}{2}\left(\hat{W}_{2}^{2}-3 \hat{W}_{3}-\hat{W}_{11}\right)$, this expressions has chances to be exponentiated. In this case, the exponent should contain even less types of operators, to provide an element from $G L(\infty)$ : it should actually be $[7,8]$ a linear combination of Casimir operators. We shall now demonstrate this.

\section{Representation via Casimir operators}

We want to find an exponential representation of the operator $\hat{O}(u)$, and what we know is that the eigenvalues of $\log \hat{O}(u)$ are logarithms of (1.8). More precisely, we need the $1 / N$-expansion of

$$
\log \left(\frac{D_{R}(N)}{N^{|R|} \cdot d_{R}}\right)=\sum_{(i, j) \in R} \log \left(1+\frac{i-j}{N}\right)=\sum_{m=1}^{\infty} \frac{(-)^{m+1}}{N^{m} \cdot m} \tilde{\sigma}_{R}(m+1)
$$


where

$$
\tilde{\sigma}_{R}(m+1)=\sum_{(i, j) \in R}(i-j)^{m}=\sum_{k=0}^{m} \frac{m !}{k !(m-k) !} \sum_{j=1}^{l(\Delta)}\left((-j)^{m-k} \sum_{i=1}^{r_{j}} i^{k}\right)
$$

In fact, one can easily check that these quantities are linear combinations of the eigenvalues $\sigma(m)$ of the Casimir operators [65],

$$
\hat{C}_{m} \chi_{R}=\sigma_{R}(m) \chi_{R}
$$

which are given by

$$
\sigma_{R}(m)=\frac{1}{m} \sum_{j=1}^{l(R)}\left(\left(r_{j}-j+1 / 2\right)^{m}-(-j+1 / 2)^{m}\right)
$$

In particular,

$$
\begin{aligned}
& \sigma_{R}(1)=\sum_{i} r_{j}=\sum_{(i, j) \in R} 1=\tilde{\sigma}_{R}(1), \\
& \sigma_{R}(2)=\frac{1}{2} \sum_{j=1}^{l(R)} r_{j}\left(r_{j}-2 j+1\right)=\sum_{j=1}^{l(R)}\left(\frac{r_{j}\left(r_{j}+1\right)}{2}-j r_{j}\right)=\sum_{(i, j) \in R}(i-j)=\tilde{\sigma}_{R}(2),
\end{aligned}
$$

However, for higher $m$ relations are a little more involved:

$$
\tilde{\sigma}_{R}(m)=\sigma_{R}(m)-\sum_{k=1} \frac{(m-1) !}{(2 k) !(m-1-2 k) !}\left(1-2^{1-2 k}\right) B_{2 k} \cdot \sigma_{R}(m-2 k)
$$

The sum has finite number of items, $k<\frac{m}{2}$, and $B_{2 k}$ are the Bernoulli numbers,

$$
\sum_{n} \frac{B_{m} t^{m}}{m !}=\frac{t e^{t}}{e^{t}-1}, \quad \text { or } \quad \sum_{n} \frac{B_{2 m} t^{2 m}}{(2 m) !}=\frac{t e^{t}}{e^{t}-1}-1-\frac{t}{2}
$$

$B_{1}=\frac{1}{2}, B_{2}=\frac{1}{6}, B_{4}=-\frac{1}{30}, B_{6}=\frac{1}{42}, B_{8}=-\frac{1}{30}, B_{10}=\frac{5}{66}, B_{12}=-\frac{691}{2730}, B_{14}=$ $\frac{7}{6}, B_{16}=-\frac{3617}{510}, \ldots$

What is important about the Casimir operators is that they contain single sums over $j$, and this property guarantees integrability [7,8]. It is of course preserved by linear combinations, i.e. $\hat{\widetilde{C}}_{n}$ with the eigenvalues $\tilde{\sigma}(n)$ are as good from this point of view as $\hat{C}_{n}$ with the eigenvalues $\sigma(n)$.

Thus we obtained the desired exponential representation of the operators

$$
\hat{O}(u)=u^{\hat{L}_{0}} \exp \left\{\sum_{m=1}^{\infty} \frac{(-)^{m+1}}{u^{m} \cdot m} \hat{\widetilde{C}}_{m+1}\right\}
$$

Moreover, when there are many $u$ variables, one can simply consider them as the Miwa-like reparametrisation of a new type of variables,

$$
\eta_{m}=\frac{(-)^{m+1}}{m} \sum_{i=1}^{n} u_{i}^{-m}, \quad \eta_{0}=\sum_{i=1}^{n} \log u_{i}
$$


and the function (2.7) becomes also a function of these additional time-variables $\eta$ :

$$
Z_{(1, n)}\left(s, u_{1}, \ldots, u_{n} \mid p\right)=\left(\prod_{i=1}^{n} \hat{O}\left(u_{i}\right)\right) e^{s p_{1}}=\exp \left(\sum_{m=0}^{\infty} \eta_{m} \hat{\widetilde{C}}_{m+1}\right) \cdot e^{s p_{1}}
$$

This function, as a function of the variables $\eta_{k}$, is very similar to the $\tau$-function $[7,8]$ :

$$
Z=\exp \left(\sum_{m=0}^{\infty} \bar{\eta}_{m} \hat{C}_{m+1}\right) \cdot e^{s p_{1}}
$$

where the variables

$$
\bar{\eta}_{m}=\sum_{i=1}^{n} u_{i}^{-m}
$$

related to $\sigma_{R}(m)$, are the linearly transformed variables $\eta_{m}$. In spite of this similarity, two functions are not connected with each other by a relation, describing equivalent integrable hierarchies [66-68]. In particular, change of the basis (3.6), which relates the operators $\hat{C}_{k}$ with $\hat{\tilde{C}}_{k}$, is not given by a change of the spectral parameter, see e.g. [69] for more details.

Explicit relation between (3.8) and (2.8) is an interesting exercise, concerning commutative algebra of cut-and-join operators and their relation to the Casimir operators. It can be easily checked in the lowest orders of the $u^{-1}$-expansion with the help of multiplication table from $[5,6]$.

\section{$4 \quad Z_{(2, n)}$ as a $\tau$-function of Toda lattice}

Eq. (3.8) immediately implies that $Z_{(2, n)}$ is a Toda lattice $\tau$-function (thus, $Z_{(1, n)}$ is a $\operatorname{KP} \tau$ function). Indeed, according to $[7,8]$ the exponential of linear combinations of the Casimir operators belongs to $G L(\infty)$ which preserves the $\mathrm{KP} /$ Toda integrability. In case of $Z_{(2, n)}$ the product of the $G L(\infty)$ operators (3.8) acts on the trivial $\tau$-function $\exp \left(\sum_{m} \frac{s^{m} p_{m} \bar{p}_{m}}{m}\right)$.

Still, there are many other ways to demonstrate that $Z_{(2, n)}$ is a $\tau$-function of the Toda lattice hierarchy. The most important is the free-fermion approach of [70] and closely related determinant formulas, see [45-51, 71]. From the point of view of Hurwitz theory, the basic well-known fact is that the character expansion

$$
\sum_{R} g_{R} \chi_{R}(p)
$$

is a KP $\tau$-function iff coefficients $g_{R}$ satisfy the Plücker relations, of which the generic solution is

$$
g_{R}=\operatorname{det}_{i j}\left(F\left(r_{i}-i, j\right)\right)
$$

with arbitrary function $F$ of two variables.

Likewise, according to [72]

$$
\tau_{n}(p, \bar{p} \mid f)=\sum_{R, R^{\prime}} f_{R, R^{\prime}}(n) \chi_{R}(p) \chi_{R^{\prime}}(\bar{p})
$$


is a Toda lattice $\tau$-function, iff

$$
f_{R, R^{\prime}}(n)=\operatorname{det}_{i j \leq n}\left(F\left(r_{i}-i, r_{j}^{\prime}-j\right)\right)
$$

Parameter $n$ here plays a role of the Toda zero-time $p_{0}$.

A particular class of solutions of this type is provided by a much simpler diagonal coefficients $f_{R, R^{\prime}}(n)[1,12,13]$

$$
f_{R, R^{\prime}}(u)=\delta_{R, R^{\prime}} \prod_{i, j \in R} f(u+i-j)
$$

where $f(x)$ is an arbitrary function of a single variable. This class of $\tau$-functions of the Toda lattice hierarchy explicitly given by the free-fermion average

$$
\tau_{n}(p, \bar{p} \mid f)=\left\langle n\left|e^{H(p)} e^{\sum T_{m}: \psi_{m}^{*} \psi_{m}:} e^{\bar{H}(\bar{p})}\right| n\right\rangle
$$

where the normal ordering is defined w.r.t. the zero vacuum: : $\psi_{m}^{*} \psi_{m}:=\psi_{m}^{*} \psi_{m}-<$ $0\left|\psi_{m}^{*} \psi_{m}\right| 0>$ and the coefficients $T_{k}$ are introduced via $f(k)=e^{T_{k-1}-T_{k}}$ with $T_{-1}=0$. More explanations of the notation see in $[12,13,70,71]$. This $\tau$-function was named hypergeometric in $[12,13]$. In particular, from $(3.6)$ is follows that the operators $\hat{O}(u)$ yield the coefficients precisely of the this form, thus the functions $Z_{(2, n)}$ belong to this class.

In fact, one can even restrict the sum in (4.3) to the diagrams with no more than $n$ lines, where $n$ is the zero-time:

$$
\tilde{\tau}_{n}(p, \bar{p} \mid f)=\sum_{R: l(R) \leq n} f_{R}(n) \chi_{R}(p) \chi_{R}(\bar{p})
$$

it is still a Toda lattice $\tau$-function $[7,8]$.

The generic Hurwitz $\tau$-function

$$
h\left(p^{(1)}, \ldots, p^{(k)} \mid \beta\right)=\exp \left(\sum_{\Delta} \beta_{\Delta} \hat{W}_{\Delta}\right) \sum_{R} d_{R}^{2-k} \chi_{R}\left\{p^{(1)}\right\} \ldots \chi_{R}\left\{p^{(k)}\right\}
$$

does not satisfy criteria (4.2) and (4.4) as a function of any time or time pairs, see $[7,8]$ for a detailed consideration (it is not even clear if it fits into the wide class of the non-Abelian $\tau$-functions of [73-76]). Notable exceptions are the cases when $k=1,2$ and when $\beta_{\Delta}$ are adjusted to provide any linear combination of the standard Casimir operators (3.4), which are nicknamed as complete cycles in [77, 78]. The functions (1.11) use additional freedom (4.5) to enlarge $k$, but keeping $p^{(3)}, \ldots, p^{(k)}$ very special: constant. This corresponds to choosing $f(x)=\prod_{i=1}^{k}\left(x+u_{i}\right)$ in (4.5) while the $s$-dependence is introduced by the rescaling $p_{k} \rightarrow s^{k} p_{k}$.

Of course, this $Z_{(2, n)}\left(u_{1}, \ldots, u_{n} \mid p, \bar{p}\right)$ is a very special kind of a lattice $\tau$-function. In particular, it possesses a simple integral representation in the form of eigenvalue matrix model (as foreseen already in $[12,13]$ ). We construct such representations in the generic case in the next section, and then consider particular more explicit examples. 


\section{$5 \quad$ Matrix model representations}

Making use of orthogonality condition $\left[79\right.$, eq. (3.1)], ${ }^{3}$

$$
\iint_{N \times N} \chi_{R}[X] \chi_{Q}[Y] e^{i \operatorname{Tr} X Y} d X d Y=\frac{D_{R}(N)}{d_{R}} \delta_{R, Q}
$$

one can easily rewrite (1.11) in the form of multi-matrix models. Indeed, from (5.6) it follows that

$$
\begin{aligned}
Z_{(2,1)}(N \mid p, \bar{p}) & =\sum_{R} \frac{D_{R}(N)}{d_{R}} \chi_{R}\{p\} \chi_{R}\{\bar{p}\} \\
& =\iint_{N \times N}\left(\sum_{R} \chi_{R}[X] \chi_{R}\{p\}\right)\left(\sum_{Q} \chi_{Q}[Y] \chi_{Q}\{\bar{p}\}\right) e^{i \operatorname{Tr} X Y} d X d Y= \\
& =\iint_{N \times N} e^{\sum_{n} \frac{1}{n} p_{n} \operatorname{Tr} X^{n}+\sum_{n} \frac{1}{n} \bar{p}_{n} \operatorname{Tr} Y^{n}} e^{i \operatorname{Tr} X Y} d X d Y
\end{aligned}
$$

what is just the conventional 2-matrix model, as was already noted in $[12,13]$.

${ }^{3}$ The simplest way to prove (5.6) is to make use of formula from Fourier theory

$$
\int d x d y f(x) g(y) e^{-x y}=\left.f\left(\frac{\partial}{\partial x}\right) g(x)\right|_{x=0}
$$

where the $x$-integral goes over the real axis, and the $y$-integral runs over the imaginary one. Now after performing the integration over angular variables and using the Itzykson-Zuber formula, one obtains the multiple eigenvalue integral

$$
\int d X d Y \chi_{R}(X) \chi_{Q}(Y) e^{-\operatorname{tr} X Y} \sim \int \prod_{i} \operatorname{det}_{i j} x_{i}^{N+R_{j}-j} \operatorname{det}_{i j} y_{i}^{N+Q_{j}-j} e^{-\sum_{i} x_{i} y_{i}}
$$

where we used the Weyl formula for the characters of linear groups

$$
\chi_{R}=\frac{\operatorname{det}_{i j} x_{i}^{N+R_{j}-j}}{\Delta(x)}
$$

and $\Delta(x)$ is the Van-der-Monde determinant. Using now formula (5.1) and

$$
\int \operatorname{det}_{i j} f_{i}\left(x_{j}\right) \operatorname{det}_{i j} g_{i}\left(y_{j}\right) \prod_{i} K\left(x_{i}, y_{i}\right)=\operatorname{det}_{i j} \int f_{i}(x) g_{j}(y) K(x, y)
$$

one immediately obtains (5.6).

This formula can be also described in the pure combinatorics terms using the Feynman diagrams. The role of propagator here is played by $\left\langle X_{i j} Y_{k l}\right\rangle=\delta_{i l} \delta_{j k}$. Therefore, the formula reduces to trivial combinatorics: connecting the free ends of multi-linear combinations of trace operators. For example,

$$
\begin{aligned}
\langle\operatorname{Tr} X \operatorname{Tr} Y\rangle & =\delta^{i j} \delta^{k l} \delta_{i l} \delta_{j k}=N, \\
\left\langle\operatorname{Tr} X^{2} \operatorname{Tr} Y^{2}\right\rangle & =2 N^{2}, \\
\left\langle\operatorname{Tr} X^{2}(\operatorname{Tr} Y)^{2}\right\rangle & =2 N, \\
\left\langle(\operatorname{Tr} X)^{2}(\operatorname{Tr} Y)^{2}\right\rangle & =2 N^{2},
\end{aligned}
$$

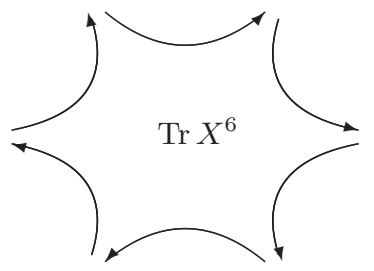


Here we used the relation

$$
\sum_{Q} \chi_{Q}[Y] \chi_{Q}\{\bar{p}\}=e^{\sum_{n} \frac{1}{n} \bar{p}_{n} \operatorname{Tr} Y^{n}}
$$

which we also need below in the form

$$
\sum_{S} \chi_{S}\left[Y_{1}\right] \chi_{S}\left[X_{2}\right]=e^{\sum_{n} \frac{1}{n} \operatorname{Tr} Y_{1}^{n} \operatorname{Tr} X_{2}^{n}}=\operatorname{Det}\left(I \otimes I-Y_{1} \otimes X_{2}\right)^{-1}
$$

Similarly to (5.7),

$$
\begin{aligned}
Z_{(2,2)} & \left(N_{1}, N_{2} \mid p, \bar{p}\right)=\sum_{R} \frac{D_{R}\left(N_{1}\right) D_{R}\left(N_{2}\right)}{d_{R}^{2}} \chi_{R}\{p\} \chi_{R}\{\bar{p}\}= \\
= & \sum_{S} \iint_{N_{1} \times N_{1}}\left(\sum_{R} \chi_{R}\{p\} \chi_{R}\left[X_{1}\right]\right) \chi_{S}\left[Y_{1}\right] e^{i \operatorname{Tr} X_{1} Y_{1}} d X_{1} d Y_{1} \\
& \times \iint_{N_{2} \times N_{2}} \chi_{S}\left[X_{2}\right]\left(\sum_{Q} \chi_{Q}\left[Y_{2}\right] \chi_{Q}\{p\}\right) e^{i \operatorname{Tr} X_{2} Y_{2}} d X_{2} d Y_{2}= \\
= & \iint_{N_{1} \times N_{1}} e^{\sum_{n} \frac{1}{n} p_{n} \operatorname{Tr} X_{1}^{n}} e^{i \operatorname{Tr} X_{1} Y_{1}} d X_{1} d Y_{1} \\
& \times \iint_{N_{2} \times N_{2}} e^{\sum_{n} \frac{1}{n} \bar{p}_{n} \operatorname{Tr} Y_{2}^{n}} e^{i \operatorname{Tr} X_{2} Y_{2}} d X_{2} d Y_{2} \frac{1}{\operatorname{Det}\left(I_{N_{1}} \otimes I_{N_{2}}-Y_{1} \otimes X_{2}\right)}= \\
= & \int_{N_{1} \times N_{1}} \int_{N_{2} \times N_{2}} d \mathcal{K}_{N_{1}}\left(Y_{1} \mid p\right) \frac{1}{\operatorname{Det}\left(I_{N_{1}} \otimes I_{N_{2}}-Y_{1} \otimes X_{2}\right)} d \mathcal{K}_{N_{2}}\left(X_{2} \mid \bar{p}\right)
\end{aligned}
$$

where generalized Kontsevich measure is defined as [80-82]

$$
d \mathcal{K}_{N}(Y \mid p)=d Y \int_{N \times N} e^{\sum_{n} \frac{1}{n} p_{n} \operatorname{Tr} X^{n}} e^{i \operatorname{Tr} X Y} d X
$$

Further,

$$
\begin{aligned}
& Z_{(2,3)}\left(N_{1}, N_{2}, N_{3} \mid p, \bar{p}\right)= \\
& =\int d \mathcal{K}_{N_{1}}\left(Y_{1} \mid p\right) \frac{1}{\operatorname{Det}\left(I_{N_{1}} \otimes I_{N_{2}}-Y_{1} \otimes X_{2}\right)} e^{i \operatorname{Tr} X_{2} Y_{2}} d X_{2} d Y_{2} \frac{1}{\operatorname{Det}\left(I_{N_{2}} \otimes I_{N_{3}}-Y_{2} \otimes X_{3}\right)} d \mathcal{K}_{N_{3}}\left(X_{3} \mid \bar{p}\right)
\end{aligned}
$$

and for generic $k$ we have:

$$
Z_{(2, n)}\left(N_{1}, \ldots, N_{n} \mid p, \bar{p}\right)=\int d \mathcal{K}_{N_{1}}\left(Y_{1} \mid p\right) \frac{\prod_{i=2}^{n-1} e^{i \operatorname{Tr} X_{i} Y_{i}} d X_{i} d Y_{i}}{\prod_{i=1}^{n-1} \operatorname{Det}\left(I_{N_{i}} \otimes I_{N_{i+1}}-Y_{i} \otimes X_{i+1}\right)} d \mathcal{K}_{N_{n}}\left(X_{n} \mid \bar{p}\right)
$$

One can observe amusing parallels with the conformal matrix models [83-85], which already have a number of other interesting applications [86-95].

One can make the Miwa transformation of time variables $p_{m}=\operatorname{Tr} \Lambda^{-m}$ in order to transform these matrix integrals to an equivalent form depending on the external matrix $\Lambda$. Sometimes it turns out very convenient as we shall see below. 


\section{Miwa transformation to Kontsevich matrix models}

Now we make the Miwa transformation of one set of the time variables in $Z_{(2, k)}$ in order to obtain matrix integrals of the Kontsevich type. This kind of integrals are sometimes more convenient. In particular, the Virasoro constraints for $Z_{(1,2)}$ are evident in this representation.

For the sake of simplicity, we consider only $Z_{(2,1)}$ case, a generic case is treated in full analogy. Thus, we make the Miwa transformation of times $\bar{p}_{m}=\operatorname{Tr} \Lambda^{-m}$ in the formula (5.7), so that

$$
\exp \left(\sum_{n} \frac{\bar{p}_{m}}{m} \operatorname{Tr} Y^{m}\right)=\frac{(\operatorname{det} \Lambda)^{N}}{\operatorname{det}(\Lambda \otimes I-I \otimes Y)}
$$

Then, the integral becomes

$$
\begin{aligned}
Z_{(2,1)}(N \mid p, \bar{p}) & =\int d X d Y e^{i \operatorname{Tr} X Y} e^{\sum_{m} \frac{p_{m}}{m} \operatorname{Tr} X^{m}} e^{\sum_{m} \frac{\bar{p} m}{m} \operatorname{Tr} Y^{m}} \\
& =\int d X d Y e^{i \operatorname{Tr} X Y} e^{\sum_{m} \frac{p_{m}}{m} \operatorname{Tr} X^{m}} \frac{(\operatorname{det} \Lambda)^{N}}{\operatorname{det}(\Lambda \otimes I-I \otimes Y)}
\end{aligned}
$$

The integral over matrix $Y$ can be easily calculated (to this end, one has first to perform integration over the angular variables and then make Fourier transform w.r.t. to the eigenvalues of $Y$ ), the result reads

$$
Z_{(2,1)}(N \mid p, \bar{p})=(-\operatorname{det} \Lambda)^{N} \int_{X_{+}} d X_{N \times N} e^{-\operatorname{Tr} X \Lambda+\sum_{m} \frac{p_{m}}{m} \operatorname{Tr} X^{m}}
$$

where integral runs over $N \times N$ positive-definite matrices, that is matrices with positive eigenvalues. This follows from the standard Fourier transform:

$$
\int \frac{e^{i x y}}{y-i 0} d y=2 \pi i \theta(x)
$$

Integral (6.3) is not yet quite of Kontsevich type: it essentially depends on the matrix size $N$ and one can not reach an arbitrary point in the space of time variables. In order to lift this restriction, one can add the logarithmic term which makes the parameter $u$ and the number of integrations independent variables:

$$
Z_{(2,1)}(u \mid p, \bar{p})=(-\operatorname{det} \Lambda)^{u} \int_{X_{+}} d X_{N \times N} e^{-\operatorname{Tr} X \Lambda+(u-N) \operatorname{Tr} \log X+\sum_{m} \frac{p_{m}}{m} \operatorname{Tr} X^{m}}
$$

One can easily check for concrete $N$ that expansion of this integral into $p_{k}$-series coincides with $Z_{(2,1)}(u \mid p, \bar{p})$ from (1.11). Note also that this integral, if considered as a function of 
time variables $\bar{p}_{m}=\operatorname{Tr} \Lambda^{-m}$, does not depend ${ }^{4}$ on $N$, which is the necessary property of Kontsevich integrals [80-82].

Integral (6.5) was obtained in [3] within a different approach. From this formula one immediately obtains a one-matrix model describing $Z_{(1,2)}(u, v \mid p)$ at integer points $v=N$. This can be done in different ways.

One possibility is to put $p_{m}=v$, then we obtain the double-logarithm model of [3]:

$$
(-\operatorname{det} \Lambda)^{u} \int_{X_{+}} d X_{N \times N} e^{-\operatorname{Tr} X \Lambda+(u-N) \operatorname{Tr} \log X-v \log (1-X)}
$$

This kind of models were thoroughly investigated in [96], still in a moment we will see that (6.6) is equivalent to an even better studied theory.

Another possibility just to put $\Lambda=1$. Then the result is

$$
\left.Z_{(1,2)}(u, v \mid p)\right|_{v=N}=\int_{X_{+}} d X_{N \times N} e^{-\operatorname{Tr} X+(u-N) \operatorname{Tr} \log X+\sum_{n} \frac{p_{n}}{n} \operatorname{Tr} X^{n}}
$$

Since this integral goes over only the positive $X_{+}$, it is equivalent to the model of complex matrices where $X$ is an obviously positive-definite matrix product $H H^{\dagger}$ [97-99]:

$$
Z^{\mathbf{C}}=\int d H d H^{\dagger} e^{\operatorname{Tr} V\left(H H^{\dagger}\right)} \sim \int \prod_{i} d h_{i}^{2} \Delta^{2}\left(h_{i}^{2}\right) e^{\sum_{i} V\left(h_{i}^{2}\right)}
$$

where $V(X)$ is arbitrary potential of the matrix model, $h_{i}^{2}$ are eigenvalues of $H H^{\dagger}$ and $\Delta(h)$ is the Van-der-Monde determinant. Thus, $Z_{(1,2)}(u, v \mid p)$ from [2-4], and, hence, the double-logarithm model (6.6) is nothing but the well-known complex matrix model. Among other things, this immediately implies the Virasoro constraints for $Z_{(1,2)}(u, v \mid p)$.

In a similar way one can make the Miwa transformation of one set of times and perform integration like (6.4) in order to obtain from (5.12) a two-matrix model representation of $Z_{1,3}$ :

$$
\begin{gathered}
\left.Z_{1,3}(u, v, w \mid p)\right|_{u=N} \sim \int_{X_{+}} d X_{N \times N} \int_{Y_{+}} d Y_{N \times N} \exp \left(-\operatorname{Tr} Y^{-1}-i \operatorname{Tr} X Y+\sum_{m} \frac{p_{m}}{m} \operatorname{Tr} X^{m}+\right. \\
+(v-N) \operatorname{Tr} \log X+(v-w-N) \operatorname{Tr} \log Y)
\end{gathered}
$$

${ }^{4}$ This integral is independent of $N$ in the following sense. Calculate the coefficient in front of, say, $p_{1} p_{2}$ at different values of $N$ :

$$
\begin{array}{ll}
N=1: & \frac{u(u+1)(u+2)}{2 \lambda^{3}} \\
N=2: & \frac{u(u+1)(u+2)}{2}\left(\frac{1}{\lambda_{1}}+\frac{1}{\lambda_{2}}\right)\left(\frac{1}{\lambda_{1}^{2}}+\frac{1}{\lambda_{2}^{2}}\right) \\
N=3: & u^{2}\left(\frac{1}{\lambda_{1}^{3}}+\frac{1}{\lambda_{2}^{3}}+\frac{1}{\lambda_{3}^{3}}\right)+\frac{u\left(u^{2}+2\right)}{2}\left(\frac{1}{\lambda_{1}}+\frac{1}{\lambda_{2}}+\frac{1}{\lambda_{3}}\right)\left(\frac{1}{\lambda_{1}^{2}}+\frac{1}{\lambda_{2}^{2}}+\frac{1}{\lambda_{3}^{2}}\right)+\frac{u^{2}}{2}\left(\frac{1}{\lambda_{1}}+\frac{1}{\lambda_{2}}+\frac{1}{\lambda_{3}}\right)^{3}
\end{array}
$$

All these expressions look different and depending on $N$, but in fact are all equal to the independent on $N$ polynomial

$$
u^{2} \bar{p}_{3}+\frac{u\left(u^{2}+2\right)}{2} \bar{p}_{1} \bar{p}_{2}+\frac{u^{2}}{2} \bar{p}_{1}^{3} .
$$


where we assume that $N \leq v \leq w$ (hence the asymmetry of the integral w.r.t. interchanging $v$ and $w)$, otherwise the integrals diverge. From experience in $[100,101]$ and $[102]$ it comes with no surprise that this $Z_{(1,3)}$ satisfies $\widetilde{W}^{(3)}$ constraints. In these constraints the values of $u, v$ and $w$ are arbitrary, and the symmetry is restored (see (7.7)).

\section{$7 \quad$ Virasoro $/ \widetilde{W}$ constraints}

The simplest way to obtain Virasoro/W constraints for $Z_{(k, n)}$ is to construct the loop equations (Ward identities) of the corresponding matrix models, which are associated with arbitrary changes of integration variables in the matrix integral. The Ward identities for the two-matrix model describing $Z_{(2, n)}$ are quite involved and are expressed in terms of the $\widetilde{W}_{\infty}$-algebra of refs. [100, 101]. However, when one set of times is eliminated things simplify a lot. In particular, when only $l$ first $\bar{p}_{i}, i \leq l$, are non-vanishing, the constraints imposed on $p$-dependence involve only $\widetilde{W}^{(i)}$-operators with $i \leq l[100,101]$. As we now see, the same seems true for $Z_{(1, n)}$ models, where all $\bar{p}$ are non-vanishing, but the same. This result can imply additional kinds of matrix-model representations for $Z_{(1, n)}$.

To begin with, $Z_{(1,1)}(u \mid p)=\exp \left(\sum_{m=1}^{\infty} \frac{u s^{m} p_{n}}{m}\right)$ satisfies

$$
\left(\hat{J}_{m}^{\mathbf{C}}-\frac{m+1}{s} \frac{\partial}{\partial p_{m+1}}\right) Z_{(1,1)}(u \mid p)=0, \quad m \geq 0
$$

with

$$
\hat{J}_{m}^{\mathbf{C}}=m \frac{\partial}{\partial p_{m}}
$$

The next model $Z_{(1,2)}$ is equivalent to the complex one-matrix model (6.7), for which the Ward identities are just the Virasoro constraints, derived in [99]:

$$
\left(\hat{L}_{m}^{\mathbf{C}}-\frac{m+1}{s} \frac{\partial}{\partial p_{m+1}}\right) Z_{(1,2)}(u, v \mid p)=0, \quad m \geq 0
$$

where

$$
\hat{L}_{m}^{\mathbf{C}}=\sum_{k=1}^{\infty}(m+k) p_{k} \frac{\partial}{\partial p_{m+k}}+\sum_{a=1}^{n-1} a(n-a) \frac{\partial^{2}}{\partial p_{a} \partial p_{m-a}}+(u+v) m \frac{\partial}{\partial p_{m}}+u v \delta_{m, 0}
$$

One can easily check that these constraints are indeed satisfied by (1.11) at $k=1, n=2$. Note that integration domain $x>0$ is preserved by the transformation $\delta x=x^{m+1}$ only for $m \geq 0$, thus there is no $\hat{L}_{-1}^{\mathrm{C}}$ constraint - this seems not to match the claim of [3]. Let us stress that in case of (7.3) the second term in the brackets can be interpreted as the shift of the $p_{1}$-variable, but this is no longer so for more general $\widetilde{W}$-constraints, see (7.1) and [100-102]. Note also that we do not include $\partial / \partial p_{0}$ terms in the sum, and give the corresponding contributions explicitly. Usually they would be proportional to the matrix size $N$, but in Virasoro constraints this size does not need to be integer. Moreover, the would be $N^{2}$ is substituted by $u v$, while $2 N$ by $(u+v)$. 
Likewise, the $Z_{(1,3)}$ function (6.9) satisfies the $\widetilde{W}^{(3)}$ constraint:

$$
\left(\hat{M}_{m}^{\mathbf{C}}-\frac{m+1}{s} \frac{\partial}{\partial p_{m+1}}\right) Z_{(1,3)}(u, v, w \mid p)=0, \quad m \geq 0
$$

where

$$
\hat{M}_{0}^{\mathbf{C}}=\sum_{a, b=1}^{\infty}\left((a+b) p_{a} p_{b} \frac{\partial}{\partial p_{a+b}}+a b p_{a+b} \frac{\partial^{2}}{\partial p_{a} \partial p_{b}}\right)+(u+v+w) \sum_{a=1}^{\infty} a p_{a} \frac{\partial}{\partial p_{a}}+u v w
$$

and, more generally,

$$
\begin{aligned}
\hat{M}_{m}^{\mathbf{C}}= & \sum_{k, l=1}^{\infty}(k+l+m) p_{k} p_{l} \frac{\partial}{\partial p_{k+l+m}}+\sum_{k=1}^{\infty}\left(\sum_{a=1}^{k+m-1}+\sum_{a=1}^{m}\right) a(k+m-a) p_{k} \frac{\partial^{2}}{\partial p_{a} \partial p_{k+m-a}}+ \\
& +\sum_{a+b+c=m} a b c \frac{\partial^{3}}{\partial p_{a} \partial p_{b} \partial p_{c}}+u v w \delta_{m, 0}+\frac{m^{2}(m+1)}{2} \frac{\partial}{\partial p_{m}}+(u v+v w+w u) m \frac{\partial}{\partial p_{m}}+ \\
& +(u+v+w)\left(\sum_{k=1}^{\infty}(k+m) p_{k} \frac{\partial}{\partial k+m}+\sum_{a+b=m} \frac{\partial^{2}}{\partial p_{a} \partial p_{b}}\right)
\end{aligned}
$$

Clearly, this time $N^{3} \longrightarrow u v w, 3 N^{2} \longrightarrow(u v+v w+w u)$ and $3 N \longrightarrow(u+v+w)$. We keep the same label $\mathbf{C}$ for these operators, to emphasize similarity with (7.4). In fact they belong to the class of the $\widetilde{W}$-operators [45-51, 100-102], appearing in description of Kontsevich and multi-matrix models and mnemonically are powers of the current $\hat{J}^{\mathbf{C}}$ defined by (9.22), subjected to peculiar normal ordering, when all the $\hat{J}_{-}^{\mathrm{C}}$ operators on the right are simply thrown away, see $[100,101]$ for a detailed description.

Similarly, one can treat the models $Z_{(1, n)}$ with higher $n>3$. They satisfy similar $\widetilde{W}^{(n)}$-constraints. In principle, they can be derived either from multi-matrix models or from any of the $W$-representations, described in the present paper.

For illustrative purposes we provide just one more example:

$$
\left(\hat{N}_{m}^{\mathbf{C}}-\frac{m+1}{s} \frac{\partial}{\partial p_{m+1}}\right) Z_{(1,4)}(u, v, w, x \mid p)=0, \quad m \geq 0
$$

and the simplest of operators $\widetilde{W}^{(4)}$ is

$$
\begin{aligned}
\hat{N}_{0}^{\mathbf{C}}= & \sum_{a, b, c=1}^{\infty}\left((a+b+c) p_{a} p_{b} p_{c} \frac{\partial}{\partial p_{a+b+c}}+a b c p_{a+b+c} \frac{\partial^{2}}{\partial p_{a} \partial p_{b} \partial p_{c}}\right)+ \\
& +\frac{3}{2} \sum_{a+b=c+d} c d p_{a} p_{b} \frac{\partial^{2}}{\partial p_{c} \partial p_{d}}+\frac{1}{2} \sum_{a, b=1}^{\infty} a b p_{a} p_{b} \frac{\partial^{2}}{\partial p_{a} \partial p_{b}}+ \\
& +(u+v+w+x) \sum_{a, b=1}^{\infty}\left((a+b) p_{a} p_{b} \frac{\partial}{\partial p_{a+b}}+a b p_{a+b} \frac{\partial^{2}}{\partial p_{a} \partial p_{b}}\right) \\
& +(u v+u w+u x+v w+v x+w x) \sum_{k=1}^{\infty} k p_{k} \frac{\partial}{\partial p_{k}}+ \\
& +\sum_{k=1}^{\infty} \frac{k^{2}(k+1)}{2} p_{k} \frac{\partial}{\partial p_{k}}+u v w x
\end{aligned}
$$




\section{Naive $W$-representations}

In addition to $W$-representation (3.8) in terms of the Casimir operators, which immediately implies integrability, one can rewrite the generating functions (2.7) as an exponential in a more straightforward way, which also provides nice expressions manifestly belonging to integrability-preserving $G L(\infty)$ group [70, 103, 104].

\subsection{The case of $Z_{(1,1)}$}

From (2.7) and from the fact that the operator $\hat{O}(u)$ in $(2.8)$ preserves unity, $\hat{O}(u) \cdot 1=1$, it follows that

$$
Z_{(1,1)}(s, u)=\hat{O}(u) \circ e^{s p_{1}} \cdot 1=\exp \left(\hat{O}(u) \circ s p_{1} \circ \hat{O}(u)^{-1}\right) \cdot 1
$$

(the last equality holds for any function, not obligatory exponential, but $Z_{(1,1)}(s, u)$ is expressed via exponential). Note that to use these kind of formulas one needs to rewrite (2.1) and (2.7) as some operator relations using composition $\circ$ instead of action of operators, i.e. $e^{s p_{1}}$ in (8.1) is treated not as a function, but as an operator (of multiplication by $e^{s p_{1}}$ ). For example, for $\hat{W}_{[1]}=\hat{L}_{0}=\sum_{n} n p_{n} \frac{\partial}{\partial p_{n}}$ and $\chi_{[1]}=p_{1}$ one has

$$
\hat{W}_{[1]} \circ \chi_{[1]}=\chi_{[1]}+\chi_{[1]} \circ \hat{W}_{[1]}
$$

and (2.1) is reproduced if we apply this identity to unity, which is annihilated by $\hat{W}_{\Delta}$ :

$$
\hat{W}_{[1]} \circ \chi_{[1]} \cdot 1=\chi_{[1]} \cdot 1+\chi_{[1]} \circ \hat{W}_{[1]} \cdot 1=\chi_{[1]} \cdot 1=p_{1}
$$

For the sake of brevity, we omit the sign of composition o throughout this section, since it is implied at any operator expressions here.

We can now use $(2.8)$ to calculate the operator $\hat{O}(u) s p_{1} \hat{O}(u)^{-1}$, which stands in the exponent in (8.1). For this we need the explicit formulas for $\hat{W}_{\Delta}$ from $[5,6]$. For $\Delta=\delta_{1} \geq$ $\delta_{2} \geq \ldots \geq \delta_{l(\Delta)} \geq 0=\{\ldots, \underbrace{2, \ldots, 2}_{m_{2}}, \underbrace{1, \ldots, 1}_{m_{1}}\}$

$$
\hat{W}_{\Delta}=\prod_{k} \frac{1}{m_{k} ! k^{m_{k}}}: \hat{D}_{k}^{m_{k}}:
$$

where $\hat{D}$ are defined in terms of the Miwa matrix $X$ from $p_{k}=\operatorname{Tr} X^{k}$ :

$$
\hat{D}_{k}=\operatorname{Tr}\left(X \frac{\partial}{\partial X^{t r}}\right)^{k}=\operatorname{Tr}\left(X \partial_{X}\right)^{k}
$$

and the double dots denote normal ordering: all the $X$-derivatives stand to the right of all $X$ 's, e.g.

$$
: \operatorname{Tr}\left(X \partial_{X}\right)^{2}:=: X_{i j} \frac{\partial}{\partial X_{k j}} X_{k l} \frac{\partial}{\partial X_{i l}}:=X_{i j} X_{k l} \frac{\partial^{2}}{\partial X_{k j} X_{i l}}
$$

(this example illustrates also the meaning of the transposition superscript $X^{t r}$ ). It is because of the normal ordering that $\hat{W}_{\Delta}$ annihilates unity. 
Now we can act with $\hat{W}_{\Delta}$ on $p_{1}$. The commutator

$$
\left[: \hat{D}_{k}:, p_{1}\right]=k: \operatorname{Tr} X^{2} \partial_{X}\left(X \partial_{X}\right)^{k-2}:
$$

This implies that

$$
\begin{aligned}
\hat{W}_{2} p_{1} & =\frac{1}{2}: \hat{D}_{2}: p_{1}=p_{1} \hat{W}_{2}+\underline{\operatorname{Tr} X^{2} \partial_{X}} \\
\hat{W}_{3} p_{1} & =\frac{1}{3}: \hat{D}_{3}: p_{1}=p_{1} \hat{W}_{3}+: \operatorname{Tr} X^{2} \partial_{X} X \partial_{X}: \\
\hat{W}_{22} p_{1} & =\frac{1}{8}:\left(\hat{D}_{2}\right)^{2}: p_{1}=p_{1} \hat{W}_{22}+\frac{1}{2}: \hat{D}_{2} \operatorname{Tr} X^{2} \partial_{X}:
\end{aligned}
$$

Now, add the two last lines:

$$
\begin{aligned}
{\left[\left(\hat{W}_{3}+\hat{W}_{22}\right), p_{1}\right] } & =\frac{1}{2}\left(: \hat{D}_{2} \operatorname{Tr} X^{2} \partial_{X}:+2: \operatorname{Tr} X^{2} \partial_{X} X \partial_{X}:\right) \\
& =\frac{1}{2} \operatorname{Tr} X^{2} \partial_{X}: \hat{D}_{2}:=\left(\underline{\operatorname{Tr} X^{2} \partial_{X}}\right) \hat{W}_{2}
\end{aligned}
$$

where the underlined operator is just the same as in the first line of (8.8).

Coming back to (8.1), we see that

$$
\begin{aligned}
\hat{O}(u) p_{1} & =\left(1+\frac{\hat{W}_{2}}{u}+\frac{\hat{W}_{3}+\hat{W}_{22}}{u^{2}}+\ldots\right) u^{\hat{L}_{0}} p_{1}=\left(u+\hat{W}_{2}+\frac{\hat{W}_{3}+\hat{W}_{22}}{u}+\ldots\right) p_{1} u^{\hat{L}_{0}}= \\
& =\left\{p_{1}\left(u+\hat{W}_{2}+\frac{\hat{W}_{3}+\hat{W}_{22}}{u}+\ldots\right)+\underline{\operatorname{Tr} X^{2} \partial_{X}}+\underline{\operatorname{Tr} X^{2} \partial_{X}} \frac{\hat{W}_{2}}{u}+\ldots\right\} u^{\hat{L}_{0}}= \\
& =u p_{1} \hat{O}(u)+\left(\underline{\operatorname{Tr} X^{2} \partial_{X}}\right) \hat{O}(u)=\left(u p_{1}+\hat{L}_{-1}\right) \hat{O}(u)
\end{aligned}
$$

where

$$
\begin{aligned}
\hat{L}_{0} & =\hat{W}_{[1]}=\operatorname{Tr} X \partial_{X}=\sum_{m} m p_{m} \frac{\partial}{\partial p_{m}}, \\
\hat{L}_{-1} & =\underline{\operatorname{Tr} X^{2} \partial_{X}}=\sum_{m} m p_{m+1} \frac{\partial}{\partial p_{m}}
\end{aligned}
$$

Thus we obtain from (8.1) a $W$-representation

$$
Z_{(1,1)}(s, u \mid p)=e^{s\left(\hat{L}_{-1}+u p_{1}\right)} \cdot 1
$$

alternative to (3.8).

\subsection{Direct check of (8.12)}

In fact, $Z_{(1,1)}(s, u \mid p)$ is known explicitly, see (1.14). The relation

$$
e^{s\left(\hat{L}_{-1}+u p_{1}\right)} \cdot 1=\exp \left(u \sum_{m} \frac{s^{m} p_{m}}{m}\right)=Z_{(1,1)}(s, u \mid p)=\sum_{R} s^{|R|} D_{R}(u) \chi_{R}\{p\}
$$


implied by (8.12), follows from the Campbell-Hausdorff formula, if it is written in the form

$$
\exp \left(\frac{[B, A]}{2}-\frac{[A,[A, B]]}{3}+\frac{[[A, B], B]}{6}+\ldots\right) \cdot e^{A} \cdot e^{B}=e^{A+B}
$$

We choose $A=\sup _{1}$ and $B=s \hat{L}_{-1}$, since in this case $e^{B} \cdot 1=1$. Then only the first and the third terms at the very left exponential contributes giving $u s^{2} p_{2} / 2$ and $u s^{3} p_{3} / 3$. More generally, only the terms of the form

$$
\sum_{m} \frac{\operatorname{ad}_{B}^{m}(A)}{m(m+1)}
$$

contribute. Since clearly $\operatorname{ad}_{B}^{m}(A)=m p_{m+1}$, while all other commutators (like $\sum_{m} \frac{\operatorname{ad}_{A}^{m}(B)}{m+1}$ ) are vanishing,

$$
\begin{aligned}
e^{s\left(\hat{L}_{-1}+u p_{1}\right)} \cdot 1 & =e^{A+B} \cdot 1=\exp \left(\sum_{m=1} \frac{\operatorname{ad}_{B}^{m}(A)}{m(m+1)}\right) e^{A} \\
& =\exp \left(\sum_{m=1} \frac{s^{m+1} u p_{m+1}}{m+1}\right) \cdot e^{s u p_{1}}=\exp \left(u \sum_{m=1} \frac{s^{m} p_{m}}{m}\right)
\end{aligned}
$$

which is exactly (8.13).

\subsection{The case of $Z_{(1,2)}$}

This time instead of (8.1) one needs

$$
Z_{(1,2)}(s, u, v)=\hat{O}(v) \hat{O}(u) e^{s p_{1}} \cdot 1=\exp \left(\hat{O}(v) \hat{O}(u) s p_{1} \hat{O}(u)^{-1} \hat{O}(v)^{-1}\right) \cdot 1
$$

and thus an appropriate modification of (8.10):

$$
\begin{aligned}
& \hat{O}(v) \hat{O}(u) p_{1}=\hat{O}(v)\left(u p_{1} \hat{O}(u)+\left(\underline{\operatorname{Tr} X^{2} \partial_{X}}\right) \hat{O}(u)\right)= \\
& =u v p_{1} \hat{O}(v) \hat{O}(u)+u\left(\underline{\operatorname{Tr} X^{2} \partial_{X}}\right) \hat{O}(v) \hat{O}(u)+\hat{O}(v)\left(\underline{\operatorname{Tr} X^{2} \partial_{X}}\right) \hat{O}(u)= \\
& =u v p_{1} \hat{O}(v) \hat{O}(u)+(u+v)\left(\underline{\operatorname{Tr} X^{2} \partial_{X}}\right) \hat{O}(v) \hat{O}(u) \\
& -\left[\frac{\operatorname{Tr} X^{2} \partial_{X}}{},\left(v+\hat{W}_{2}+\frac{\hat{W}_{3}+\hat{W}_{22}}{v}+\ldots\right)\right] \hat{O}(u)= \\
& =u v p_{1} \hat{O}(v) \hat{O}(u)+(u+v)\left(\underline{\operatorname{Tr} X^{2} \partial_{X}}\right) \hat{O}(v) \hat{O}(u) \\
& +\left(\underline{\underline{\operatorname{Tr} X^{2} \partial_{X} X \partial_{X}:}}\right) \hat{O}(v) \hat{O}(u)= \\
& =\left(u v p_{1}+(u+v) \hat{L}_{-1}+\hat{M}_{-1}\right) \hat{O}(v) \hat{O}(u)
\end{aligned}
$$

with

$$
\begin{aligned}
& \hat{L}_{-1}=\underline{\operatorname{Tr} X^{2} \partial_{X}}=\sum_{m} m p_{m+1} \frac{\partial}{\partial p_{m}} \\
& \hat{M}_{-1}=\underline{\underline{\operatorname{Tr} X^{2} \partial_{X} X \partial_{X}:}}=\sum_{a, b}(a+b-1) p_{a} p_{b} \frac{\partial}{\partial p_{a+b-1}}+a b p_{a+b+1} \frac{\partial^{2}}{\partial p_{a} \partial_{b}}
\end{aligned}
$$


Combining this with (8.17) we immediately reproduce the result of [2]:

$$
Z_{(1,2)}(s, u, v)=\exp \left\{s\left(u v p_{1}+(u+v) \hat{L}_{-1}+\hat{M}_{-1}\right)\right\} \cdot 1
$$

\subsection{Operators $\hat{\mathcal{O}}\left(u_{1}, \ldots, u_{n}\right)$}

Now generalizing (8.17), one can define the operator $\hat{\mathcal{O}}\left(u_{1}, \ldots, u_{n}\right)$

$$
Z_{(1, n)}\left(s, u_{1}, \ldots, u_{n}\right)=\exp \left(\prod_{i=1}^{k} \hat{O}\left(u_{i}\right) s p_{1} \prod_{I=1}^{n} \hat{O}\left(u_{i}\right)^{-1}\right) \cdot 1=\hat{\mathcal{O}}\left(u_{1}, \ldots, u_{n}\right) \cdot 1
$$

The sequence of underlined operators is evidently

$$
\operatorname{ad}_{\hat{W}_{2}}^{k} p_{1}=: \operatorname{Tr} X^{2} \partial_{X}\left(X \partial_{X}\right)^{k-1}:
$$

in particular,

$$
\begin{aligned}
\hat{L}_{-1} & =\left[\hat{W}_{2}, p_{1}\right]=\operatorname{Tr} X^{2} \partial_{X} \\
\hat{M}_{-1} & =\left[\hat{W}_{2}, \operatorname{Tr} X^{2} \partial_{X}\right]=: \operatorname{Tr} X^{2} \partial_{X} X \partial_{X}: \\
\hat{N}_{-1} & =\left[\hat{W}_{2},: \operatorname{Tr} X^{2} \partial_{X} X \partial_{X}:\right]=: \operatorname{Tr} X^{2} \partial_{X}\left(X \partial_{X}\right)^{2}:
\end{aligned}
$$

Therefore the naive $W$-representations of the functions $Z_{k}$ look as follows:

$$
Z_{(1, k)}(\vec{u})=\hat{\mathcal{O}}_{k}(\vec{u}) \cdot 1
$$

where

$$
\begin{aligned}
\hat{\mathcal{O}}_{1} & =e^{s p_{1}} \\
\hat{\mathcal{O}}_{2}(u) & =e^{s\left(\hat{L}_{-1}+u p_{1}\right)}, \\
\hat{\mathcal{O}}_{3}(u, v) & \stackrel{[2]}{=} e^{s\left(\hat{M}_{-1}+(u+v) \hat{L}_{-1}+u v p_{1}\right)}, \\
\hat{\mathcal{O}}_{4}(u, v, w) & =e^{s\left(\hat{N}_{-1}+(u+v+w) \hat{M}_{-1}+(u v+v w+w u) \hat{L}_{-1}+u v w p_{1}\right)},
\end{aligned}
$$

and

$$
\begin{aligned}
\hat{L}_{-1}= & \sum_{m} m p_{m+1} \frac{\partial}{\partial p_{m}} \\
\hat{M}_{-1}= & \sum_{a, b}(a+b-1) p_{a} p_{b} \frac{\partial}{\partial p_{a+b-1}}+a b p_{a+b+1} \frac{\partial^{2}}{\partial p_{a} \partial_{b}}, \\
\hat{N}_{-1}= & \sum_{a, b, c=1}^{\infty}\left((a+b+c-1) p_{a} p_{b} p_{c} \frac{\partial}{\partial p_{a+b+c-1}}+a b c p_{a+b+c+1} \frac{\partial^{3}}{\partial p_{a} \partial p_{b} \partial p_{c}}\right)+ \\
& +\frac{3}{2} \sum_{a, b=1}^{\infty} \sum_{c=1}^{a+b} a b p_{c} p_{a+b+1-c} \frac{\partial^{2}}{\partial p_{a} \partial p_{b}}+\frac{1}{2} \sum_{a=1}^{\infty} a^{2}(a+1) p_{a+1} \frac{\partial}{\partial p_{a}},
\end{aligned}
$$

Formula (8.20) for $Z_{(1,2)}$ appeared in [2].

Note that this representation of the operators $\hat{\mathcal{O}}_{k}(\vec{u})$ also makes manifest that they are elements of $G L(\infty)[70,103,104]$ which gives yet another proof of integrability: this property guarantees that $Z_{(1, n)}(\vec{u})$ is a $\tau$-function of the KP hierarchy. 


\subsection{Hierarchy in $n$}

Operators (8.25) form a clear hierarchy in $n$, and one can easily move in $n$ in both directions. Let us look at the simpler one: the decrease of $n$.

Since $D_{R}(v)=d_{R} v^{|R|}\left(1+O\left(v^{-1}\right)\right)$, one has

$$
\lim _{v \longrightarrow \infty} Z_{(1, n+1)}\left(\frac{s}{v}, \vec{u}, v\right)=Z_{(1, n)}(s, \vec{u})
$$

For example, for $n=0$,

$$
\lim _{v \longrightarrow \infty} \exp \left(\sum_{m} \frac{(s / v)^{m} \cdot v}{m} p_{m}\right)=e^{s p_{1}}
$$

Thus

$$
\hat{\mathcal{O}}_{n}(\vec{u})=\lim _{v \longrightarrow \infty} \hat{\mathcal{O}}_{n+1}(v, \vec{u})^{1 / v}
$$

In particular, taking $\hat{\mathcal{O}}_{2}$ from [2], we immediately get:

$$
\ldots \longrightarrow \exp \left\{s\left(\hat{M}_{-1}+(u+v) \hat{L}_{-1}+u v p_{1}\right)\right\} \longrightarrow \exp \left\{s\left(\hat{L}_{-1}+u p_{1}\right)\right\} \longrightarrow e^{s p_{1}}
$$

It now looks rather obvious that the previous term on the left is

$$
\exp \left\{s\left(\hat{N}_{-1}+(u+v+w) \hat{M}_{-1}+(u v+v w+w u) \hat{L}_{-1}+u v w p_{1}\right)\right\}
$$

and so on.

\section{Description in terms of the $w_{\infty}$-algebra}

The $W$-representation (3.8) can be further transformed and simplified. Since it is expressed through the Casimir operators (3.4), which belong to the $W_{\infty}$ algebra, and no central extensions are relevant for our considerations, one can make use of its alternative representation in terms of ordinary differential operators [105]. This is a very powerful technique, see [69] for the recent review, and this also turns to be the case in application to our problem.

\subsection{Combined Casimir operators $\hat{\widetilde{C}}$ as distinguished $\hat{W}_{0}^{(m)}$}

In this approach operators from $w_{\infty}$ are represented by polynomial of $z$ and $D=z \partial_{z}$. In most considerations $D$ can be considered just as an integer number. In particular, the standard Casimir operators (3.4) are mapped [69, 105] into

$$
\hat{C}(n) \longrightarrow \frac{\left(D-\frac{1}{2}\right)^{n}-\left(-\frac{1}{2}\right)^{n}}{n}
$$

Substituting this into the sums in (3.6), we obtain that combined Casimir operators, given by this seemingly complicated formula, are in fact mapped into something clearly distinguished:

$$
\hat{\widetilde{C}}(n+1) \longrightarrow \sum_{i=1}^{D-1} i^{n}
$$


and then, from (3.8)

$$
\hat{O}(u)=u^{\hat{C}_{1}} \exp \left\{\sum_{n=1}^{\infty} \frac{(-)^{n+1}}{u^{n} \cdot n} \hat{\widetilde{C}}(n+1)\right\} \rightarrow u^{D} \exp \left(\sum_{i=0}^{D-1} \log \left(1+\frac{i}{u}\right)\right)=\frac{\Gamma(u+D)}{\Gamma(u)}
$$

i.e. as an element of the $w_{\infty}$ algebra, operator $\hat{O}(u)$ is just an ordinary $\Gamma$-function! In fact, Bernoulli numbers naturally arise in the coefficients of the large- $u$ asymptotics of $\log \Gamma(u)$.

Moreover, the sums at the r.h.s. (9.2) are also associated with the very special operators, what provides a spectacular interpretation of $\hat{\widetilde{C}}(n)$. Namely, monomials $z D^{n}$ are images of

$$
\begin{aligned}
p_{1} & \longrightarrow z \cdot 1, \\
\hat{L}_{-1}=\sum_{n} n p_{n+1} \frac{\partial}{\partial p_{n}} & \longrightarrow z \cdot D, \\
\hat{M}_{-1}=\sum_{a, b}\left((a+b-1) p_{a} p_{b} \frac{\partial}{\partial p_{a+b-1}}+a b p_{a+b+1} \frac{\partial^{2}}{\partial p_{a} \partial p_{b}}\right) & \longrightarrow z \cdot D^{2},
\end{aligned}
$$

and the sums in (9.2) are the zeroth harmonics of the same operators:

$$
\begin{aligned}
\hat{L}_{0}=\sum_{n} n p_{n+1} \frac{\partial}{\partial p_{n}} & \longrightarrow D=\sum_{i=1}^{D-1} 1, \\
\hat{M}_{0}=\sum_{a, b}\left((a+b) p_{a} p_{b} \frac{\partial}{\partial p_{a+b}}+a b p_{a+b} \frac{\partial^{2}}{\partial p_{a} \partial p_{b}}\right) & \longrightarrow D(D-1)=2 \sum_{i=1}^{D-1} i, \\
\hat{N}_{0}=\sum_{a, b, c=1}^{\infty}\left((a+b+c) p_{a} p_{b} p_{c} \frac{\partial}{\partial p_{a+b+c}}+a b c p_{a+b+c} \frac{\partial^{3}}{\partial p_{a} \partial p_{b} \partial p_{c}}\right)+ & \\
+\frac{3}{2} \sum_{a, b=1}^{\infty} \sum_{c=1}^{a+b-1} a b p_{c} p_{a+b-c} \frac{\partial^{2}}{\partial p_{a} \partial p_{b}}+\frac{1}{2} \sum_{a=1}^{\infty} a\left(a^{2}-1\right) p_{a} \frac{\partial}{\partial p_{a}} & \longrightarrow \frac{1}{2} D(D-1)(2 D-1)=3 \sum_{i=1}^{D-1} i^{2}
\end{aligned}
$$

Let us introduce a unified notation $\hat{W}_{n}^{(m)}$ for all these $W$-operators:

$$
p_{k}=\hat{W}_{k}^{(1)}, \quad \hat{L}_{k}=\hat{W}_{k}^{(2)}, \quad \hat{M}_{k}=\hat{W}_{k}^{(3)}, \quad \hat{N}_{k}=\hat{W}_{k}^{(4)}, \ldots
$$

Comparing (9.2) with (9.5) we see that

$$
\hat{\widetilde{C}}(n)=\frac{1}{n} \hat{W}_{0}^{(n+1)}
$$

In terms of these operators one can rewrite (3.8) and (9.3) as

$$
\hat{O}(u)=\exp \left(\log u \hat{L}_{0}+\frac{1}{2 u} \hat{M}_{0}-\frac{1}{6 u^{2}} \hat{N}_{0}+\ldots\right)=\exp \left(\sum_{m=2}^{\infty} \frac{(-)^{m} \hat{W}_{0}^{(m+1)}}{(m-1) m u^{m-1}}\right) u^{\hat{W}_{0}^{(2)}}
$$

so that

$$
Z_{(1, n)}(s, \vec{u})=\prod_{i=1}^{n} \hat{O}\left(u_{i}\right) \cdot e^{s p_{1}}=\exp \left(\sum_{m=2}^{\infty} \eta_{m} \hat{W}_{0}^{(m+1)}\right) \cdot \exp \left(s p_{1} \prod_{i=1}^{n} u_{i}\right)
$$


with

$$
\eta_{m}=\frac{(-)^{m}}{(m-1) m} \sum_{i=1}^{n} \frac{1}{u_{i}^{m-1}}
$$

\subsection{Relation between the two $W$-representations}

At the same time, from (8.25) the same function is given by

$$
\begin{aligned}
Z_{1, n}(s, \vec{u}) & =\exp \left(s u_{1} \ldots u_{n}\left\{p_{1}+\sum_{i=1}^{n} \frac{1}{u_{i}} \hat{L}_{-1}+\sum_{i<j}^{n} \frac{1}{u_{i} u_{j}} \hat{M}_{-1}+\sum_{i<j<k}^{n} \frac{1}{u_{i} u_{j} u_{k}} \hat{N}_{-1}+\ldots\right\}\right) \cdot 1= \\
& =\exp \left(s\left(\prod_{i=1}^{n} u_{i}\right)\left(\sum_{m=0}^{\infty} \xi_{m} \hat{W}_{-1}^{(m+1)}\right)\right) \cdot 1
\end{aligned}
$$

with

$$
\xi_{m}=\sum_{i_{1} \leq i_{2} \leq \ldots \leq i_{m}} \frac{1}{u_{i_{1}} u_{i_{2}} \ldots u_{i_{m}}}
$$

In this form there are two differences between (9.9) and (9.11): the grading of $\hat{W}$-operators ( 0 and -1 respectively) and the time variables $\eta$ and $\xi$, given respectively by power sum and elementary symmetric polynomials of variables $u_{i}^{-1}$.

These two $W$-representations are of course related by the Campbell-Hausdorff formula, this time in the form

$$
e^{\hat{B}} e^{\hat{A}}=e^{\hat{A}+[\hat{B}, \hat{A}]+\frac{1}{2 !}[\hat{B}[\hat{B}, \hat{A}]]+\ldots} e^{\hat{B}}
$$

when exponent in the boxed operator is just

$$
\hat{C}=\sum_{m=0}^{\infty} \frac{1}{m !} \operatorname{ad}_{\hat{B}}^{m} \hat{A}
$$

where we need to substitute $\hat{A}=p_{1}$ and $\hat{B}=\sum_{m} \eta_{m} \hat{W}_{0}^{(m)}$. Since (9.14) is linear in $\hat{A}$, the common factor $s \prod u_{i}$ can be omitted and restored at the very end. Then, if applied to unity, the l.h.s. of (9.13) gives (9.9), and the r.h.s. will provide (9.11), because $e^{\hat{B}} \cdot 1=1$. To calculate $\hat{C}$ we need a commutation relation

$$
\left[\hat{W}_{0}^{(m+1)}, \hat{W}_{-1}^{(n+1)}\right]=m \hat{W}_{-1}^{m+n}
$$

which provides $\hat{C}$ in the following form:

$\hat{C}=\underbrace{\hat{W}_{-1}^{(1}}_{p_{1}}+\sum_{m=2}^{\infty} m \eta_{m} \hat{W}_{-1}^{(m)}+\frac{1}{2 !} \sum_{m, n=2}^{\infty} m n \eta_{m} \eta_{n} \hat{W}_{-1}^{(m+n)}+\frac{1}{2 !} \sum_{l, m, n=2}^{\infty} l m n \eta_{l} \eta_{m} \eta_{n} \hat{W}_{-1}^{(l+m+n)}+\ldots$ 
We want this to be equal to $\sum_{k=0}^{\infty} \xi_{k} \hat{W}_{-1}^{(k+1)} \quad$ Clearly, each $\xi_{k}$ is a finite multi-linear combination of $\eta_{m}$, for example,

$$
\begin{aligned}
& \xi_{0}=1 \\
& \xi_{1}=2 \eta_{2}=\sum_{i} \frac{1}{u_{i}} \\
& \xi_{2}=3 \eta_{3}+2 \eta_{2}^{2}=-\frac{1}{2} \sum_{i} \frac{1}{u_{i}^{2}}+\frac{1}{2}\left(\sum_{i} \frac{1}{u_{i}}\right)^{2}=\sum_{i<j} \frac{1}{u_{i} u_{j}}, \\
& \xi_{3}=4 \eta_{4}+6 \eta_{2} \eta_{3}+\frac{4}{3} \eta_{2}^{2}=\sum_{i<j<k} \frac{1}{u_{i} u_{j} u_{k}},
\end{aligned}
$$

Thus (9.9) and (9.11) - and thus (3.8) and (8.25) - are indeed related by the simplest of all Campbell-Hausdorff formulas (9.13).

\subsection{More details from the $w_{\infty}$ dictionary}

Higher harmonics of the simplest operators $\hat{W}^{(m)}$ are mapped into the following polynomials of $z$ and $D=z \partial_{z}$ :

$$
\begin{aligned}
\hat{J}_{k}=\operatorname{res}_{z}\left(z^{k} \hat{J}(z)\right) & \longrightarrow j_{k}=z^{-k}, \quad k \neq 1 \\
\hat{L}_{k}=\frac{1}{2} \operatorname{res}_{z}\left(z^{1+k}: \hat{J}(z)^{2}:\right) & \longrightarrow l_{k}=z^{-k}\left(z \partial_{z}-\frac{k+1}{2}\right) \\
\hat{M}_{k}=\frac{1}{3} \operatorname{res}_{z}\left(z^{2+k}: \hat{J}(z)^{3}:\right) & \longrightarrow m_{k}=z^{-k}\left(z^{2} \partial_{z}^{2}-k z \partial_{z}+\frac{(1+k)(2+k)}{6}\right), \\
\hat{N}_{0} & \longrightarrow \frac{1}{2}\left(2 z \partial_{z}-1\right)\left(z \partial_{z}-1\right) z \partial_{z}, \quad \hat{N}_{-1} \longrightarrow z\left(z \partial_{z}\right)^{3}
\end{aligned}
$$

(polynomials at the r.h.s. are defined up to constant terms, which do not affect commutators - expressions in (9.5) make use of this freedom). In general, for peculiar operators, which are made from the current

$$
\hat{J}(x)=\sum_{m} \frac{\hat{J}_{m}}{x^{m+1}}=\sum_{m=1}^{\infty}\left(p_{m} x^{m-1}+\frac{m}{x^{m+1}} \frac{\partial}{\partial p_{m}}\right)
$$

and its derivatives — and at the same time belong to the $W_{\infty}$ algebra — the mapping rule is:

$$
\operatorname{res}_{z}\left(z^{-k}: \frac{\left(\hat{J}(z)+\partial_{z}\right)^{m+1}}{m+1}: 1\right) \longrightarrow\left(z^{2} \partial_{z}\right)^{m} z^{k}
$$


It is easy to check that above examples fit into this scheme, with

$$
\begin{aligned}
\hat{L}(x) & =\sum_{m} \frac{\hat{L}_{m}}{x^{m+2}}=: \hat{J}(x)^{2}: \\
\hat{M}(x) & =\sum_{m} \frac{\hat{M}_{m}}{x^{m+3}}=: \hat{J}(x)^{3}: \\
\hat{N}(x) & =\sum_{m} \frac{\hat{N}_{m}}{x^{m+4}}=: \hat{J}(x)^{4}-\left(\partial_{x} \hat{J}(x)\right)^{2}:
\end{aligned}
$$

Note, that this formalism is applicable only to operators from $W_{\infty}$ algebra, i.e. those made from the current (9.19) and its derivatives in a very special way - as linear combinations of those at the 1.h.s. of (9.20). Already the forth power of the current, : $\hat{J}^{4}$ :, does not belong to this algebra - this is the reason for the $(\partial \hat{J})^{2}$ subtraction in $\hat{N} \in W_{\infty}$. Another typical example are Virasoro operators $\hat{L}_{n}^{\mathbf{C}}$ in (7.4). They are actually made from the square of another current,

$$
\hat{J}^{\mathbf{C}}(x)=\sum_{m=1}^{\infty}\left(\frac{1}{2} p_{m} x^{m-1}+\frac{m}{x^{m+1}} \frac{\partial}{\partial p_{m}}\right)
$$

with additional factor $1 / 2$ in the poshtive harmonics. Because of this the $w_{\infty}$ technique, described in this section, can not be used to prove and even check the Virasoro constraints (7.4): it does not adequately describe commutation relations between $\hat{L}_{n}^{\mathbf{C}} \notin W_{\infty}$ and $\hat{L}_{0}, \hat{M}_{0}, \hat{N}_{0}, \ldots \in W_{\infty}$. However, there are two amusing exceptions: the zero harmonics $\hat{L}_{0}^{\mathrm{C}}$ and $\hat{M}_{0}^{\mathrm{C}}$ do belong to $W_{\infty}$, this is no longer true neither for $\hat{N}_{0}^{\mathrm{C}}$, nor for higher harmonics of $\hat{L}^{\mathbf{C}}$ and $\hat{M}^{\mathbf{C}}$.

\section{Conclusion}

This paper gives a brief summary of existing knowledge about the simple family (1.11) with $k=1,2$. This family consists of Hurwitz $\tau$-functions which are integrable in the simplest KP/Toda sense. A number of facts are already present in the literature, not only we presented them in a systematic way revealing all the relations between these facts, but we naturally made a number of new claims:

- In addition to the naive $W$-representation in section 8 we described two others: in terms of the generalized cut-and-join operators, (2.8) and of the Casimir operators, (3.8), providing a direct relation to the Hurwitz theory a $l a[5,6]$ and to the $\mathrm{KP} /$ Toda integrability respectively. One more version, (9.8), provides a bridge between naive and Casimir $W$-representations.

- We put together the two-matrix and Kontsevich like models from $[3,12,13]$ and pointed out an intriguing relation of higher $Z_{(2, n)}$ to the conformal like matrix models. 
- We provided a description of the most studied $Z_{(1,2)}$ model in terms of complex matrix model which directly provides the Virasoro constraints, (7.4). Similarly, the $Z_{(1,3)}$ model is described by the asymmetric two-matrix model with $1 / Y$ potential and satisfies the $\widetilde{W}^{(3)}$-constraints, etc.

- We interpreted (-1)-modes of $W$-operators which enter the naive $W$-representation of [2] and its generalizations as multiple commutators of the basic pair: the cut-andjoin operator $\hat{W}_{[2]}=\frac{1}{2}: \operatorname{Tr}\left(X \partial_{X}\right)^{2}:$ and $\hat{L}_{-1}=: \operatorname{Tr}\left(X^{2} \partial_{X}\right)$ :

- We explained in section 9 how the mapping to the differential operators can be used to drastically simplify derivation of these and many other similar results (note, however, that this approach is directly applicable only to the $\mathrm{KP} /$ Toda, but not to general Hurwitz $\tau$-functions, and is thus restricted to models (1.11)).

There are still a lot of formulas to derive, especially for $Z_{(2, n)}$ models with $n>1$.

\section{Acknowledgments}

We are grateful to L.Chekhov for useful discussions. Our work is partly supported by ERC Starting Independent Researcher Grant StG No. 204757-TQFT (A.A.), the grants NSh-1500.2014.2 (A.A., A.M.'s) and NSh-5138.2014.1 (S.N.), by RFBR 13-02-00457 (A.A., A.Mir. and S.N.), 13-02-00478 (A.Mor.), by joint grants 13-02-91371-ST, 14-01-92691-Ind, by the Brazil National Counsel of Scientific and Technological Development (A.Mor.), by Laboratory of Quantum Topology of Chelyabinsk State University (Russian Federation government grant 14.Z50.31.0020) (S.N.) and by FRIAS (A.M.'s).

Open Access. This article is distributed under the terms of the Creative Commons Attribution License (CC-BY 4.0), which permits any use, distribution and reproduction in any medium, provided the original author(s) and source are credited.

\section{References}

[1] I.P. Goulden and D.M. Jackson, The KP hierarchy, branched covers, and triangulations, arXiv:0803.3980.

[2] P. Zograf, Enumeration of Grothendieck's dessins and KP hierarchy, arXiv:1312.2538.

[3] J. Ambjørn and L. Chekhov, The matrix model for dessins d'enfants, arXiv: 1404.4240 [INSPIRE].

[4] M. Kazaryan and P. Zograf, Virasoro constraints and topological recursion for Grothendieck's dessin counting, arXiv:1406.5976.

[5] A. Mironov, A. Morozov and S. Natanzon, Complete set of cut-and-join operators in Hurwitz-Kontsevich theory, Theor. Math. Phys. 166 (2011) 1 [arXiv:0904.4227] [INSPIRE].

[6] A. Mironov, A. Morozov and S. Natanzon, Algebra of differential operators associated with Young diagrams, J. Geom. Phys. 62 (2012) 148 [arXiv:1012.0433] [INSPIRE]. 
[7] S. Kharchev, A. Marshakov, A. Mironov and A. Morozov, Generalized Kazakov-Migdal-Kontsevich model: group theory aspects, Int. J. Mod. Phys. A 10 (1995) 2015 [hep-th/9312210] [InSPIRE].

[8] A. Alexandrov, A. Mironov, A. Morozov and S. Natanzon, Integrability of Hurwitz partition functions. I. Summary, J. Phys. A 45 (2012) 045209 [arXiv:1103.4100] [InSPIRE].

[9] A. Mironov, A. Morozov and A. Sleptsov, Genus expansion of HOMFLY polynomials, Theor. Math. Phys. 177 (2013) 1435 [Teor. Mat. Fiz. 177 (2013) 179] [arXiv:1303.1015] [INSPIRE].

[10] A. Mironov, A. Morozov and A. Sleptsov, On genus expansion of knot polynomials and hidden structure of Hurwitz $\tau$-functions, Eur. Phys. J. C 73 (2013) 2492 [arXiv: 1304.7499] [INSPIRE].

[11] A. Mironov, A. Morozov, A. Sleptsov and A. Smirnov, On genus expansion of superpolynomials, arXiv:1310.7622 [INSPIRE].

[12] A. Orlov and D.M. Shcherbin, Hypergeometric solutions of soliton equations, Theor. Math. Phys. 128 (2001) 906.

[13] A. Orlov, Hypergeometric functions as infinite-soliton $\tau$ functions, Theor. Math. Phys. 146 (2006) 183.

[14] R. Dijkgraaf, Mirror symmetry and elliptic curves, in The moduli spaces of curves, Progr. Math. 129 (1995) 149.

[15] D.E. Littlewood, The theory of group characters and matrix representations of groups, Clarendon Press, Oxford U.K. (1958).

[16] M. Hamermesh, Group theory and its application to physical problems, Dover, New York U.S.A. (1989).

[17] I.G. Macdonald, Symmetric functions and Hall polynomials, Oxford Science Publications, Oxford U.K. (1995).

[18] W. Fulton, Young tableaux: with applications to representation theory and geometry, London Mathematical Society, London U.K. (1997).

[19] A. Mironov, A. Morozov and S. Natanzon, Integrability properties of Hurwitz partition functions. II. Multiplication of cut-and-join operators and WDVV equations, JHEP 11 (2011) 097 [arXiv:1108.0885] [INSPIRE].

[20] A.Y. Morozov, Unitary integrals and related matrix models, Theor. Math. Phys. 162 (2010) 1 [Teor. Mat. Fiz. 161 (2010) 3] [arXiv:0906.3518] [INSPIRE].

[21] G. Belyi, On Galois extensions of a maximal cyclotomic field, Math. USSR Izvestiya 14 (1980) 247.

[22] A. Grothendieck, Sketch of a programme, Lond. Math. Soc. Lect. Note Ser. 242 (1997) 243.

[23] A. Grothendieck, Esquisse d'un programme, in Geometric Galois action, P. Lochak and L. Schneps eds., Cambridge University Press, Cambridge U.K. (1997), pg. 5.

[24] G.B. Shabat and V.A. Voevodsky, The Grothendieck Festschrift, V.III, Birkhäuser, (1990), pg. 199.

[25] S.K. Lando and A.K. Zvonkin, Graphs on surfaces and their applications, in Encycl. Math. Sci. 141, Springer, Germany (2004).

[26] C. Itzykson and J.B. Zuber, Matrix integration and combinatorics of modular groups, Commun. Math. Phys. 134 (1990) 197 [inSPIRE]. 
[27] R. de Mello Koch and S. Ramgoolam, From matrix models and quantum fields to Hurwitz space and the absolute Galois group, arXiv:1002.1634 [INSPIRE].

[28] T.W. Brown, Complex matrix model duality, Phys. Rev. D 83 (2011) 085002 [arXiv: 1009.0674] [INSPIRE].

[29] E. Witten, On the structure of the topological phase of two-dimensional gravity, Nucl. Phys. B 340 (1990) 281 [INSPIRE].

[30] M. Kontsevich, Intersection theory on the moduli space of curves, Funk. Anal. Priloz. 25 (1991) 50.

[31] A.M. Polyakov, Quantum geometry of bosonic strings, Phys. Lett. B 103 (1981) 207 [INSPIRE].

[32] A.M. Polyakov, Quantum geometry of fermionic strings, Phys. Lett. B 103 (1981) 211 [INSPIRE].

[33] A. Marshakov, A. Mironov and A. Morozov, On equivalence of topological and quantum $2 D$ gravity, Phys. Lett. B 274 (1992) 280 [hep-th/9201011] [INSPIRE].

[34] E. Witten, On the Kontsevich model and other models of two-dimensional gravity, in Proc. Differential geometric methods in theoretical physics, vol. 1, New York U.S.A. (1991), pg. 176.

[35] V.A. Kazakov, The appearance of matter fields from quantum fluctuations of $2 D$ gravity, Mod. Phys. Lett. A 4 (1989) 2125 [inSPIRE].

[36] E. Brézin and V.A. Kazakov, Exactly solvable field theories of closed strings, Phys. Lett. B 236 (1990) 144 [INSPIRE].

[37] M.R. Douglas and S.H. Shenker, Strings in less than one-dimension, Nucl. Phys. B 335 (1990) 635 [inSPIRE].

[38] D.J. Gross and A.A. Migdal, Nonperturbative two-dimensional quantum gravity, Phys. Rev. Lett. 64 (1990) 127 [INSPIRE].

[39] M.R. Douglas, Strings in less than one-dimension and the generalized $K^{-} D^{-} V$ hierarchies, Phys. Lett. B 238 (1990) 176 [INSPIRE].

[40] M. Fukuma, H. Kawai and R. Nakayama, Continuum Schwinger-Dyson equations and universal structures in two-dimensional quantum gravity, Int. J. Mod. Phys. A 6 (1991) 1385 [inSPIRE].

[41] R. Dijkgraaf, H.L. Verlinde and E.P. Verlinde, Loop equations and Virasoro constraints in nonperturbative $2 D$ quantum gravity, Nucl. Phys. B 348 (1991) 435 [INSPIRE].

[42] A. Levin and A. Morozov, On the foundations of the random lattice approach to quantum gravity, Phys. Lett. B 243 (1990) 207 [INSPIRE].

[43] R. Gopakumar, What is the simplest gauge-string duality?, arXiv:1104.2386 [INSPIRE].

[44] N. Adrianov et al., Catalog of dessins d'enfants with no more than 4 edges, J. Math. Sci. 158 (2009) 22 [arXiv:0710.2658].

[45] A. Morozov, String theory: what is it?, Sov. Phys. Usp. 35 (1992) 671.

[46] A. Morozov, Integrability and matrix models, Phys. Usp. 37 (1994) 1 [hep-th/9303139] [INSPIRE].

[47] A. Morozov, Matrix models as integrable systems, hep-th/9502091 [INSPIRE].

[48] A. Morozov, Challenges of matrix models, hep-th/0502010 [INSPIRE]. 
[49] A. Mironov, 2D gravity and matrix models. 1. 2D gravity, Int. J. Mod. Phys. A 9 (1994) 4355 [hep-th/9312212] [InSPIRE].

[50] A. Mironov, Matrix models of two-dimensional gravity, Phys. Part. Nucl. 33 (2002) 537 [Fiz. Elem. Chast. Atom. Yadra 33 (2002) 1051] [INSPIRE].

[51] A. Mironov, Matrix models vs. matrix integrals, Theor. Math. Phys. 146 (2006) 63 [Teor. Mat. Fiz. 146 (2006) 77] [hep-th/0506158] [INSPIRE].

[52] A. Morozov, Faces of matrix models, JETP Lett. 95 (2012) 586 [arXiv:1204.3953] [INSPIRE].

[53] A.S. Alexandrov, A. Mironov and A. Morozov, Partition functions of matrix models as the first special functions of string theory. 1. Finite size Hermitean one matrix model, Int. J. Mod. Phys. A 19 (2004) 4127 [Teor. Mat. Fiz. 142 (2005) 419] [hep-th/0310113] [INSPIRE].

[54] A.S. Alexandrov, A. Mironov and A. Morozov, M-theory of matrix models, Theor. Math. Phys. 150 (2007) 153 [Teor. Mat. Fiz. 150 (2007) 179] [hep-th/0605171] [INSPIRE].

[55] A.S. Alexandrov, A. Mironov and A. Morozov, Instantons and merons in matrix models, Physica D 235 (2007) 126 [hep-th/0608228] [INSPIRE].

[56] A. Alexandrov, A. Mironov and A. Morozov, BGWM as second constituent of complex matrix model, JHEP 12 (2009) 053 [arXiv:0906.3305] [INSPIRE].

[57] A.S. Alexandrov, A. Mironov, A. Morozov and P. Putrov, Partition functions of matrix models as the first special functions of string theory. II. Kontsevich model, Int. J. Mod. Phys. A 24 (2009) 4939 [arXiv:0811.2825] [inSPIRE].

[58] B. Eynard, Topological expansion for the 1-Hermitian matrix model correlation functions, JHEP 11 (2004) 031 [hep-th/0407261] [INSPIRE].

[59] L. Chekhov and B. Eynard, Hermitean matrix model free energy: Feynman graph technique for all genera, JHEP 03 (2006) 014 [hep-th/0504116] [INSPIRE].

[60] L. Chekhov and B. Eynard, Matrix eigenvalue model: Feynman graph technique for all genera, JHEP 12 (2006) 026 [math-ph/0604014] [INSPIRE].

[61] N. Orantin, Symplectic invariants, Virasoro constraints and Givental decomposition, arXiv:0808.0635 [INSPIRE].

[62] A. Morozov and S. Shakirov, Generation of matrix models by $W$-operators, JHEP 04 (2009) 064 [arXiv: 0902.2627] [INSPIRE].

[63] A. Morozov and S. Shakirov, On equivalence of two Hurwitz matrix models, Mod. Phys. Lett. A 24 (2009) 2659 [arXiv:0906.2573] [INSPIRE].

[64] A. Alexandrov, Matrix models for random partitions, Nucl. Phys. B 851 (2011) 620 [arXiv:1005.5715] [INSPIRE].

[65] D.P. Zhelobenko, Compact Lie group and their representations, American Mathematical Society, U.S.A. (1973).

[66] T. Shiota, Characterization of Jacobian varieties in terms of soliton equations, Invent. Math. 83 (1986) 333.

[67] S. Kharchev, A. Marshakov, A. Mironov and A. Morozov, Landau-Ginzburg topological theories in the framework of GKM and equivalent hierarchies, Mod. Phys. Lett. A 8 (1993) 1047 [Theor. Math. Phys. 95 (1993) 571] [Teor. Mat. Fiz. 95 (1993) 280] [hep-th/9208046] [INSPIRE]. 
[68] S. Kharchev, Kadomtsev-Petviashvili hierarchy and generalized Kontsevich model, hep-th/9810091 [INSPIRE].

[69] A. Alexandrov, Enumerative geometry, $\tau$-functions and Heisenberg-Virasoro algebra, arXiv: 1404.3402 [INSPIRE].

[70] E. Date, M. Jimbo, M. Kashiwara and T. Miwa, Transformation groups for soliton equations, in RIMS Symp. "Non-linear integrable systems - classical theory and quantum theory", World Scientific, Singapore (1983).

[71] S. Kharchev, A. Marshakov, A. Mironov and A. Morozov, Generalized Kontsevich model versus Toda hierarchy and discrete matrix models, Nucl. Phys. B 397 (1993) 339 [hep-th/9203043] [INSPIRE].

[72] K. Takasaki, Group representations and systems of differential equations, Adv. Studies Pure Math. 4 (1984) 139.

[73] A. Gerasimov, S. Khoroshkin, D. Lebedev, A. Mironov and A. Morozov, Generalized Hirota equations and representation theory. 1 . The case of $\mathrm{SL}(2)$ and $\mathrm{SL}_{q}(2)$, Int. J. Mod. Phys. A 10 (1995) 2589 [hep-th/9405011] [inSPIRE].

[74] S. Kharchev, A. Mironov and A. Morozov, Non-standard KP evolution and quantum

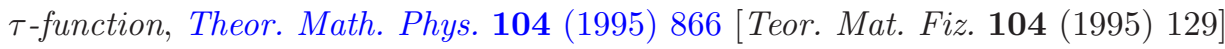
[q-alg/9501013].

[75] A. Mironov, Quantum deformations of tau functions, bilinear identities and representation theory, hep-th/9409190 [INSPIRE].

[76] $\tau$-function within group theory approach and its quantization, Theor. Math. Phys. 114 (1998) 127 [Teor. Mat. Fiz. 114 (1998) 163] [q-alg/9711006].

[77] A. Okounkov and R. Pandharipande, Gromov-Witten theory, Hurwitz theory, and completed cycles, Ann. Math. 163 (2006) 517 [math.AG/0204305].

[78] S. Lando, Combinatorial facets of Hurwitz numbers, in Applications of group theory to combinatorics, J. Koolen, J.H. Kwak and M.-Y. Xu eds., Taylor \& Francis Group, London U.K. (2008), pg. 109.

[79] S. Corley, A. Jevicki and S. Ramgoolam, Exact correlators of giant gravitons from dual $N=4$ SYM theory, Adv. Theor. Math. Phys. 5 (2002) 809 [hep-th/0111222] [InSPIRE].

[80] S. Kharchev, A. Marshakov, A. Mironov, A. Morozov and A. Zabrodin, Unification of all string models with $c<1$, Phys. Lett. B 275 (1992) 311 [hep-th/9111037] [INSPIRE].

[81] S. Kharchev, A. Marshakov, A. Mironov, A. Morozov and A. Zabrodin, Towards unified theory of 2D gravity, Nucl. Phys. B 380 (1992) 181 [hep-th/9201013] [INSPIRE].

[82] A. Mironov, A. Morozov and G.W. Semenoff, Unitary matrix integrals in the framework of generalized Kontsevich model. 1. Brezin-Gross-Witten model, Int. J. Mod. Phys. A 11 (1996) 5031 [hep-th/9404005] [InSPIRE].

[83] S. Kharchev, A. Marshakov, A. Mironov, A. Morozov and S. Pakuliak, Conformal matrix models as an alternative to conventional multimatrix models, Nucl. Phys. B 404 (1993) 717 [hep-th/9208044] [INSPIRE].

[84] A. Mironov and S. Pakulyak, On the continuum limit of the conformal matrix models, Theor. Math. Phys. 95 (1993) 604 [Teor. Mat. Fiz. 95 (1993) 317] [Int. J. Mod. Phys. A 8 (1993) 3107] [hep-th/9209100] [INSPIRE]. 
[85] H. Awata, Y. Matsuo, S. Odake and J. Shiraishi, A note on Calogero-Sutherland model, $W_{n}$ singular vectors and generalized matrix models, Soryushiron Kenkyu 91 (1995) A69 [hep-th/9503028] [INSPIRE].

[86] A. Marshakov, A. Mironov and A. Morozov, Generalized matrix models as conformal field theories: discrete case, Phys. Lett. B 265 (1991) 99 [INSPIRE].

[87] R. Dijkgraaf and C. Vafa, Toda theories, matrix models, topological strings and $N=2$ gauge systems, arXiv:0909.2453 [INSPIRE].

[88] H. Itoyama, K. Maruyoshi and T. Oota, The quiver matrix model and $2 d-4 d$ conformal connection, Prog. Theor. Phys. 123 (2010) 957 [arXiv:0911.4244] [INSPIRE].

[89] T. Eguchi and K. Maruyoshi, Penner type matrix model and Seiberg-Witten theory, JHEP 02 (2010) 022 [arXiv:0911.4797] [InSPIRE].

[90] T. Eguchi and K. Maruyoshi, Seiberg-Witten theory, matrix model and AGT relation, JHEP 07 (2010) 081 [arXiv:1006.0828] [InSPIRE].

[91] R. Schiappa and N. Wyllard, An $A_{r}$ threesome: matrix models, $2 d$ CFTs and $4 d N=2$ gauge theories, J. Math. Phys. 51 (2010) 082304 [arXiv:0911.5337] [INSPIRE].

[92] A. Mironov, A. Morozov and S. Shakirov, Matrix model conjecture for exact BS periods and Nekrasov functions, JHEP 02 (2010) 030 [arXiv:0911.5721] [INSPIRE].

[93] A. Mironov, A. Morozov and S. Shakirov, Conformal blocks as Dotsenko-Fateev integral discriminants, Int. J. Mod. Phys. A 25 (2010) 3173 [arXiv:1001.0563] [INSPIRE].

[94] A. Mironov, A. Morozov and S. Shakirov, Towards a proof of AGT conjecture by methods of matrix models, Int. J. Mod. Phys. A 27 (2012) 1230001 [arXiv:1011.5629] [InSPIRE].

[95] A. Mironov, A. Morozov and S. Shakirov, A direct proof of AGT conjecture at $\beta=1$, JHEP 02 (2011) 067 [arXiv: 1012.3137] [INSPIRE].

[96] L. Chekhov and K. Palamarchuk, Two logarithm matrix model with an external field, Mod. Phys. Lett. A 14 (1999) 2229 [hep-th/9811200] [INSPIRE].

[97] T.R. Morris, Checkered surfaces and complex matrices, Nucl. Phys. B 356 (1991) 703 [INSPIRE].

[98] Yu. Makeenko, Complex matrix model and two-dimensional quantum gravity, Pis'ma $v$ ZhETF 52 (1990) 885 [JETP Lett. 52 (1990) 259] [INSPIRE].

[99] Y. Makeenko, A. Marshakov, A. Mironov and A. Morozov, Continuum versus discrete Virasoro in one matrix models, Nucl. Phys. B 356 (1991) 574 [InSPIRE].

[100] A. Marshakov, A. Mironov and A. Morozov, From Virasoro constraints in Kontsevich's model to $W$ constraints in two matrix model, Mod. Phys. Lett. A 7 (1992) 1345 [hep-th/9201010] [INSPIRE].

[101] C.-R. Ahn and K. Shigemoto, One point functions of loops and constraints equations of the multimatrix models at finite $N$, Phys. Lett. B 285 (1992) 42 [hep-th/9112057] [INSPIRE].

[102] S. Kharchev, A. Marshakov, A. Mironov and A. Morozov, Generalized Kazakov-Migdal-Kontsevich model: group theory aspects, Int. J. Mod. Phys. A 10 (1995) 2015 [hep-th/9312210] [inSPIRE].

[103] G. Segal and G. Wilson, Loop groups and equations of KdV type, Publ. I.H.E.S. 61 (1985) 5.

[104] M. Kazarian, KP hierarchy for Hodge integrals, arXiv:0809.3263.

[105] M. Fukuma, H. Kawai and R. Nakayama, Infinite dimensional Grassmannian structure of two-dimensional quantum gravity, Commun. Math. Phys. 143 (1992) 371 [INSPIRE]. 